\title{
Asymmetric Stable Paretian Distribution Testing
}

\author{
Marc S. Paolella ${ }^{a, b *}$ \\ ${ }^{a}$ Department of Banking and Finance, University of Zurich, Switzerland \\ ${ }^{b}$ Swiss Finance Institute
}

April 2016

\begin{abstract}
Two new tests for the symmetric stable Paretian distribution with tail index $1<\alpha<2$ are proposed. The test statistics and their associated approximate $p$-values are instantly computed and do not require use of the stable density or distribution or maximum likelihood estimation. They exhibit high power against a variety of alternatives, and much higher power than the existing test based on the empirical characteristic function. The two tests are combined to yield a test that has substantially higher power. A fourth test based on likelihood ratio is also studied. Extensions are proposed to address the asymmetric case and are shown to have reasonable actual size properties and high power against several viable alternatives.
\end{abstract}

Keywords: Hill-type Estimators; Likelihood Ratio Test; Tail Index.

*Address: Plattenstrasse 14, 8032 Zurich, Switzerland. Tel: +4144 634-4584. E-mail address: marc.paolella@bf.uzh.ch. Matlab programs for the computation of the ALHADI, $\tau_{20}$, and (for sample sizes 500, 1000 and 2500) the combined $A+\tau_{20}$ tests, and their approximate $p$-values, are available as supplementary material.

(C) 2016. This manuscript version is made available under the Elsevier user license http://www.elsevier.com/open-access/userlicense/1.0/ 


\section{Introduction}

The use of the stable Paretian distribution for modeling unconditional or conditional financial asset returns has had a tenuous reception since its debut in Mandelbrot (1963) and Fama (1963). Its proponents argue that it is the only limiting distribution of sums of (properly standardized) random variables (the generalized central limit theorem), and nests the normal distribution as a special case, and so is justified from the perspective that model error terms are often viewed as embodying the sum of a large number of random variables not accounted for in the model. Furthermore, the stable distribution, as its name suggests, exhibits sum-stability, so that, for example, the portfolio distribution of a set of assets can be characterized. Finally, from a purely practical point of view, (daily) asset returns exhibit the heavy tails and asymmetry of the stable Paretian distribution. McCulloch (1997a), Rachev and Mittnik (2000), Borak et al. (2005), and Nolan (2016) offer extensive accounts of the stable distribution and its wide applicability in finance and other scientific fields, while Samorodnitsky and Taqqu (1994) provides a more technical book on the univariate and, particularly, the multivariate setting.

The critics of the stable Paretian distribution claim at least three problems (see, e.g., Jondeau et al., 2007). The first is that the stable density, being intractable, renders estimation and inference very difficult. While that was historically indeed the case, it is certainly no longer true; see the discussion below in Section 2.

The second problem sometimes brought forth is that asset returns exhibit conditional heteroskedasticity, and thus cannot be independent and identically distributed (i.i.d.) stable Paretian; see, e.g., Lau et al. (1990), Ghose and Kroner (1995), Groenendijk et al. (1995), and the references therein, on the relationship between GARCH and (symmetric) stable processes. This is correct, and can be resolved by considering not the unconditional returns - which are anyway of less interest for short-term risk prediction and asset allocation - but rather the conditional returns, where conditioning is on the time-varying volatility captured, for example, via GARCH-type processes. Mittnik et al. (2000) demonstrate that, after accounting for GARCH-type conditional volatility in daily financial return series, the assumption of a stable Paretian distribution as the underlying innovation i.i.d. sequence is tenable in some cases.

The third problem claimed is that, without a variance, it cannot be a candidate distribution, citing evidence of the existence of second moments in financial data in papers such as Loretan and Phillips (1994). However, the determination of the maximally existing moment of a set of data is difficult; see McCulloch (1997b), Mittnik et al. (1998), McNeil et al. (2005), and the references therein for detailed discussions and critique of studies which claim the existence of second (and third) moments based on use of Hill-type estimators (see below). The existence of moments is determined from the asymptotic tail behavior, but in practical applications, there are never enough data points to accurately characterize the tail - the clear discussion and examples in Heyde and Kou (2004) should settle this issue.

Another, yet weaker, argument brought forth regarding the existence of variance is that, ultimately, in all applications, the actual support of the random variable underlying the phenomenon under study is bounded. While this is certainly theoretically true, we should note that, as stated eloquently by Nolan (1999, p. 2), "Of course the same people who argue that the population is inherently bounded and therefore must have a finite variance routinely use the normal distribution — with unbounded support- 
as a model for this same population. The variance is but one measure of spread for a distribution, and it is not appropriate for all problems."

Testing the stable Paretian distribution has been considered by several authors. Koutrouvelis and Meintanis (1999), Meintanis (2005), and Matsui and Takemura (2008) use the empirical characteristic function to develop testing procedures in the symmetric stable case; see Section 3.1 below. Fama and Roll (1971), Lau and Lau (1993), and Paolella (2001) consider the change in the estimated tail index, $\alpha$, as the data are summed: If the data are stable, then the value of $\alpha$ should not change, whereas for many alternatives outside the stable class, the classic central limit theorem will be at work, and the values of $\alpha$ will tend to increase towards two as the data are summed. McCulloch and Percy (2013) propose a set of composite (unknown parameter case) tests for, among others, the symmetric stable distribution and are able to reject the null in their applications, though they attribute this primarily to the presence of asymmetry.

This paper (i) provides an improvement to the test in Paolella (2001) that results in higher power, (ii) develops a new test based on properties of the order statistics of the stable distribution, (iii) combines the two former tests to produce a test with higher power, (iv) constructs likelihood ratio tests (LRT) for the composite alternatives of Student's $t$ and noncentral Student's $t$ (NCT), and (v) extends the testing paradigm to the asymmetric stable case. The combined test and the LRTs are shown to have the best power against various alternatives.

The remainder of this paper is structured as follows. Section 2 provides notation and relevant background material. Section 3 details the new tests for the symmetric stable distribution, while Section 4 considers the asymmetric stable case. Section 5 concludes and discusses a concurrent research application. Details on the calculation of $p$-values associated with the tests are discussed in an Appendix.

\section{Notation and Background Material}

\subsection{Stable Distribution and Parameter Estimation}

Let $X$ be a location-scale symmetric stable Paretian (sometimes referred to as symmetric $\alpha$-stable, or $\mathrm{S} \alpha \mathrm{S})$ random variable, denoted $X \sim S_{\alpha}(\mu, \sigma)$, for $0<\alpha \leqslant 2, \mu \in \mathbb{R}$ and $\sigma>0$. Its distribution is most commonly expressed via its characteristic function (cf) as

$$
\phi_{X}(t ; \alpha, \mu, \sigma)=\mathbb{E}[\exp (i t X)]=\exp \left(i \mu t-|\sigma t|^{\alpha}\right)
$$

For $X$ asymmetric stable Paretian, denoted $X \sim S_{\alpha, \beta}(\mu, \sigma)$, with asymmetry parameter $\beta,-1 \leqslant \beta \leqslant 1$, its $\mathrm{cf}$, for the case relevant for us, with $1<\alpha \leqslant 2$, is most often given by

$$
\phi_{X}(t ; \alpha, \beta, \mu, \sigma)=\exp \left(i \mu t-|\sigma t|^{\alpha}\left[1-i \beta \operatorname{sgn}(t) \tan \frac{\pi \alpha}{2}\right]\right), \quad 1<\alpha \leqslant 2 .
$$

Simulation of $S_{\alpha, \beta}(\mu, \sigma)$ realizations (which is crucial for determining the distribution under the null of the test statistics considered in this paper) is conducted via the method of Chambers et al. (1976).

Approximate computation of the probability density function (pdf) of $X$, as for example required for computing the maximum likelihood estimator (MLE), has been considered by numerous authors. 
We advocate use of the method in Nolan (1997) for the symmetric case, while for the asymmetric case, the fast Fourier transform is reasonably fast and effective for $1.1<\alpha<2$ (see Mittnik et al., 1999; and Paolella, 2007, Chaps. 1 and 8 for a detailed development), though polynomial spline approximations are available for $1<\alpha \leqslant 2$, such as in Doganoglu and Mittnik (1998) and Nolan (as implemented in his stable toolbox for Matlab), and are accurate enough for typical use and by far the fastest option.

There exist other methods for estimation of the four-parameter location-scale asymmetric stable distribution in the i.i.d. case which obviate the need to evaluate the pdf. One, in particular, will be of use to us in the development of the test in Section 3.3 below, namely that of McCulloch (1986). It is trivial to compute, consistent (to the extent that the granularity of two required tables with respect to $\alpha$ and $\beta$ increases with the sample size) and applicable for $0.6<\alpha \leqslant 2$ and $-1 \leqslant \beta \leqslant 1$. Its estimator of $\alpha$ relies on the 5\%,95\%,25\%, and 75\% sample quantiles (and a table lookup operation), whereas the so-called Hint estimator (discussed in Section 2.2 below) uses a different set of order statistics to yield an unbiased, consistent estimator of $\alpha$. As such, their behaviors differ markedly when the data are not stable, and thus give rise to a distribution testing procedure.

\subsection{Nonparametic Estimation of the Tail Index}

For data coming from a distribution such that the right tail is asymptotically Pareto, the cornerstone of nonparametric inference for the tail index is the Hill estimator, introduced in Hill (1975). Various derivations of the estimator can be found in McNeil et al. (2005). It requires specification of tuning parameter $k$, which indicates "where the tail starts", and is given by

$$
1 / \hat{\alpha}_{\mathrm{Hill}}(k ; \mathbf{X})=k^{-1} \sum_{j=1}^{k} \log \left(X_{T+1-j: T}\right)-\log X_{T-k: T},
$$

where $X_{j: T}$ denotes the $j$ th order statistic of sample $\mathbf{X}=\left(X_{1}, \ldots, X_{T}\right)$. For a given sample size $T$, small values of $k$ would seem to be preferred because one is further into the right tail, but the dearth of the ensuing number of tail observations results in high inaccuracy of the estimator. This is a classic example of a bias/variance tradeoff, and attempts at choosing $k$ to minimize the mean squared error would require knowledge of the true distribution.

While not suited as an estimator of the stable tail index, it can be used to construct a trivially computed estimator with extraordinary statistical properties, in ways superior to the MLE, as discussed next. Based on the Hill estimator, Mittnik and Paolella (1999) developed a scale-invariant estimator for tail index $\alpha$ for location-zero $\mathrm{S} \alpha \mathrm{S}$ data, referred to as the Hill-intercept or Hint, estimator, and denoted as $\hat{\alpha}_{\text {Hint }}$. It is valid for (at least) $1 \leqslant \alpha \leqslant 2$ and makes use of the empirical observation that the Hill estimator is very nearly linear as a function of $k$ for stable Paretian data over a large range of $1 \leqslant \alpha \leqslant 2$, tuning parameter $k$ and sample size $T$. It was found that both the intercept and slope of this linear approximation can be used to derive estimates of $\alpha$. Using the intercept, the estimator takes the simple form

$$
\hat{\alpha}_{\text {Hint }}=\hat{\alpha}_{\text {Hint }}(\mathbf{X})=-0.8110-0.3079 \hat{b}+2.0278 \hat{b}^{0.5},
$$


where $\hat{b}$ is the intercept in the simple linear regression of $\hat{\alpha}_{\text {Hill }}(\mathbf{k} ; \operatorname{abs}(\mathbf{X}))$ on $\mathbf{k} / 1000$; the elements of $\mathbf{k}$ are such that $0.2 T \leqslant k \leqslant 0.8 T$ in steps of $\max \{[T / 100], 1\}$; and the absolute value of the data are used because we assume symmetry.

In addition to its trivial computation, even in samples as small as $T=50$, the estimator is essentially unbiased for $\alpha \in[1,2]$ and almost exactly normally distributed. Furthermore, for given sample size, the variance of $\hat{\alpha}_{\mathrm{Hint}}$ is practically constant across $\alpha$, reaching its maximum for $\alpha=1.5$. For sample sizes $50<T<10,000$, this is given approximately by

$$
\widehat{\mathrm{SE}}\left(\hat{\alpha}_{\mathrm{Hint}}\right) \approx 0.0322-0.00205 T_{*}+0.02273 T_{*}^{-1}-0.0008352 T_{*}^{-2},
$$

where $T_{*}=T / 1000$. Finally, simulations show that, for the sample sizes $50<T<10,000$, the MLE performs only slightly better in terms of mean squared error. As such, the Hint estimator will form the basis of the test for $\mathrm{S} \alpha \mathrm{S}$ data in Section 3.2 below.

\section{Testing Procedures}

We begin with the existing test based on the discrepancy of the theoretical and empirical characteristic functions in Section 3.1. We then turn to the new tests, the first one based on the summability property of stable random variables, in Section 3.2, and then the so-called ALHADI test, which is based on the difference of two consistent estimators of tail index $\alpha$, in Section 3.3. Section 3.4 illustrates how the tests can be combined to produce tests with higher power, while Section 3.5 outlines the construction of a likelihood ratio test for a composite alternative. Section 3.6 shows the actual size for a nominal size of 0.05 , for the three sample sizes $T=500,1000$ and 2500, and Section 3.7 provides power comparisons based on this nominal test size and sample sizes.

\subsection{Test Based on the Empirical Characteristic Function}

For data vector $\mathbf{X}=\left(X_{1}, \ldots, X_{T}\right)$, let $Y_{j}=\left(X_{j}-\hat{\mu}\right) / \hat{\sigma}$, where consistent estimators of $\mu$ and $\sigma$ are used, as discussed in Section 2.1. The empirical characteristic function (ecf) is given by

$$
\phi_{T}(t ; \mathbf{X})=\phi_{T}(t ; \mathbf{X} ; \hat{\mu}, \hat{\sigma})=\frac{1}{T} \sum_{j=1}^{T} \exp \left(i t Y_{j}\right) .
$$

As the cf of each $Y_{j}$, with estimated $\alpha$, is $\phi(t ; \hat{\alpha})=\exp \left(-|t|^{\hat{\alpha}}\right)$, a natural test statistic for assessing the validity of the assumed distribution is

$$
D_{T, \kappa}=D_{T, \kappa}(\mathbf{X})=T \int_{-\infty}^{\infty}\left|\phi_{T}(t ; \mathbf{X})-\phi(t ; \hat{\alpha})\right|^{2} w(t) \mathrm{d} t, \quad w(t)=\exp (-\kappa|t|), \quad \kappa>0 .
$$

Matsui and Takemura (2008) derive the asymptotic distribution of (7) under the null and, via simulation for power assessment, recommend choosing $\kappa=5$. Our goal is to develop a method that can quickly deliver an accurate approximation to the $p$-value, along with the test statistic. This is more useful in inference than only delivering, say, the binary result of a test at the $10 \%, 5 \%$ and $1 \%$ levels. Also, it allows for the construction of combined tests; see Section 3.4 below. The method of approximating 
the $p$-value is detailed in Appendix A, along with more details of the actual size obtained from this procedure.

\subsection{Summability Test and Modification}

The summability test of Paolella (2001) is designed for i.i.d. location-zero S $\alpha$ S data with tail index $1<\alpha \leqslant 2$, but is scale-invariant. As such, an estimate of $\mu$ is required. We suggest use of the McCulloch (1986) estimator, for speed and simplicity reasons, and then proceed with $\mathbf{X}=\mathbf{Y}-\hat{\mu}$. The estimator $\hat{\alpha}_{\text {Hint }}$ is used in place of the theoretical value of $\alpha$ required below.

Denote by $s$ the level of aggregation applied to data vector $\mathbf{X}=\left(X_{1}, \ldots, X_{T}\right)$, i.e., for $s=1$, the entire data vector is used; for $s=2$, the data are reduced to $\mathbf{X}_{(2)}=\left(X_{1}+X_{2}, X_{3}+X_{4}, X_{5}+X_{6}, \ldots,\right)$; for $s=3, \mathbf{X}_{(3)}=\left(X_{1}+X_{2}+X_{3}, X_{4}+X_{5}+X_{6}, \ldots,\right)$, etc., and let $\hat{\alpha}(s)=\hat{\alpha}_{\text {Hint }}(s)$ denotes the estimate of $\alpha$ based on the Hint estimator for given level of aggregation $s$. For sample size $T$, the aggregation values used are $s=1,2, \ldots,[T / 100]$, so that the last $\hat{\alpha}(s)$ is based on at least 100 observations. Under the null hypothesis of (i.i.d., symmetric) stable data, the $\hat{\alpha}(s)$ should be constant, while for non-stable i.i.d. data, they are expected to increase. Thus, we consider estimating a simple linear trend model, as a linear regression of $\hat{\alpha}(s)$ onto a constant and $s$, with the slope coefficient denoted $\hat{b}$. By using the Hill-intercept estimator with its optimal properties discussed above, each $\hat{\alpha}(s)$ can be treated as a realization from a normal distribution with known variance, so that weighted least squares can be used to compute $\hat{b}$, where the weights are inversely proportional to the standard error of $\hat{\alpha}(s)$, in $(5)$, delivered with the Hill-intercept estimator.

Consider using the studentized test statistic given by $\tau_{0}=\tau_{0}(\mathbf{X})=\hat{b} / \widehat{\mathrm{SE}}(b)$. Paolella $(2001)$ determined simple functions of $T$ and $\alpha$ to compute the cutoff values for sizes $0.01,0.05$ and 0.10 , associated with the right tail of its distribution under the null hypothesis. We now augment this procedure in two ways. The first issue to realize is that the test statistic $\tau_{0}$ (and thus the hypothesis test outcome) is not invariant to permutations of the data (though observe that $\hat{\alpha}_{\text {Hint }}$ is invariant). As the data are purported to be i.i.d., the ordering should not play a role. To alleviate this issue, we take

$$
\tau_{B}=\tau_{B}(\mathbf{X})=B^{-1} \sum_{i=1}^{B} \tau_{0}\left(\mathbf{X}^{[i]}\right),
$$

where $\mathbf{X}^{[i]}$ denotes a random permutation of the data such that all the $X_{i}$ appear once, i.e., sampling without replacement. Notice that, unless all theoretically possible permutations are used (or, taking $B=\infty$ if they are randomly drawn), the outlined procedure will still return different test statistics for the same data set (unless the set of seed values for the random permutations is held constant to some arbitrary choice). While this feature is still undesirable, it cannot be avoided with finite $B$ and random permutations.

The second augmentation is to deliver an approximate $p$-value of the $\tau_{B}$ test statistic, instead of only the test outcomes at the usual three levels of significance. The method for conducting this is given in Appendix B, as well as a study confirming that the resulting actual size is accurate enough for application at the $10 \%, 5 \%$ and $1 \%$ testing levels. 


\subsection{ALHADI: The $\alpha$-Hat Discrepancy Test}

The motivation for this new test statistic comes from the structure of the McCulloch (1986) and Hint estimators for $\alpha$. Observe that the McCulloch estimator uses only the order statistics associated with quantiles $0.95,0.05,0.75$, and 0.25 , whereas Hint uses a much larger set, in such a way as to measure the tail behavior, via the Hill estimator. One might speculate that, when applying this finding to data that are not stable Paretian, these estimates could differ substantially. This is indeed the case for several alternatives of interest. This concept of assessing the appropriateness of the stable assumption by comparing different consistent estimators of the tail index is not new; see, e.g., Nolan (1999, p. 9). Our goal is to formalize the process and develop a test that is (as nearly as possible) size-correct and, with it, a $p$-value.

First consider the distributions of the McCulloch and Hint estimators under the null hypothesis. Figure 1 shows, via boxplots, the behavior of these two estimators, as well as the MLE, for S $\alpha \mathrm{S}$ data using four values of $\alpha$ and the two sample sizes $T=250$ and $T=2500$ (and based on 1000 replications). The symmetry of $\hat{\alpha}_{\text {Hint }}$, as well as its unbiasedness and nearly same variance as the MLE, is apparent.
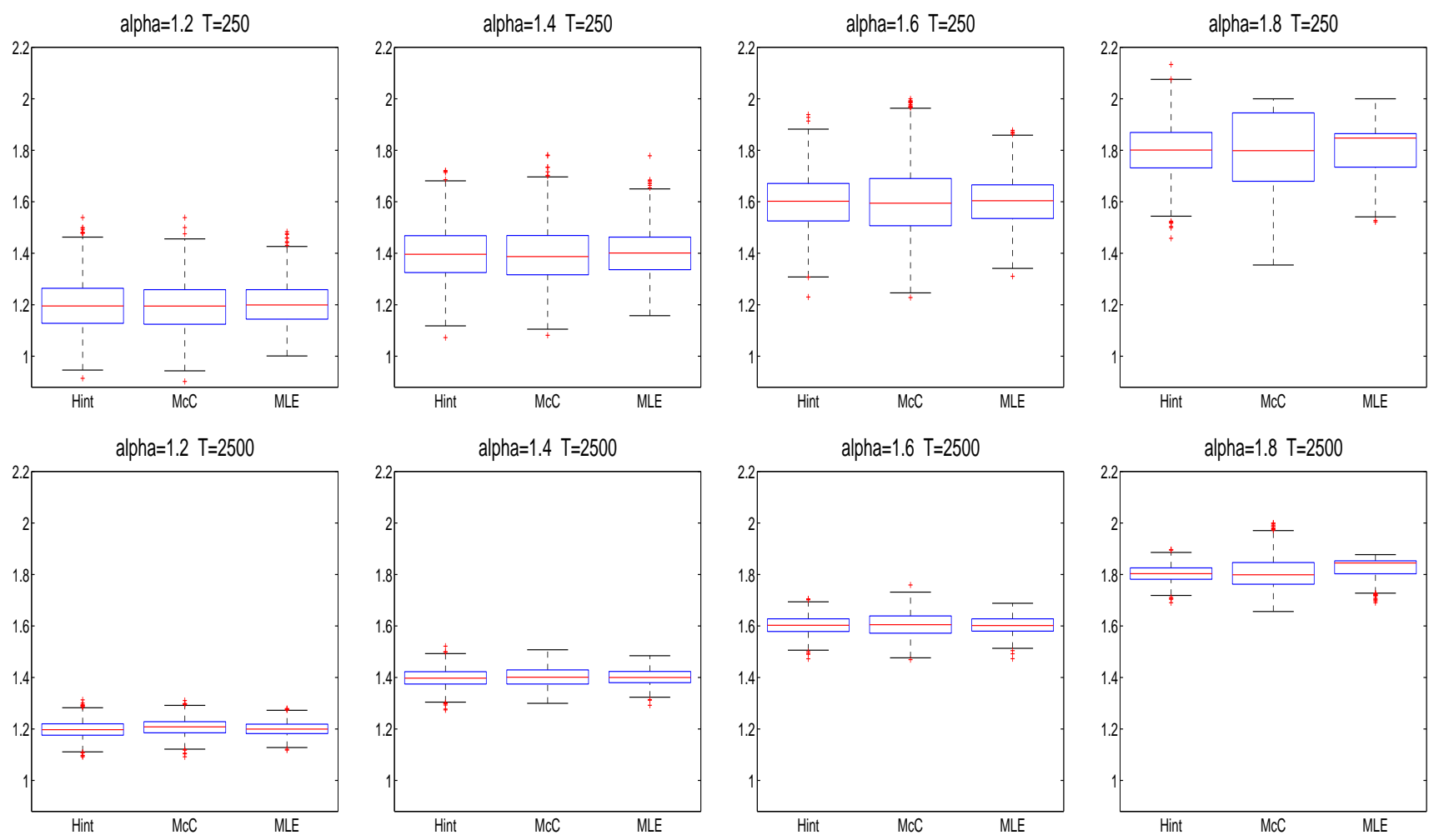

Figure 1: Boxplots of $\hat{\alpha}_{\mathrm{Hint}}, \hat{\alpha}_{\mathrm{McC}}$ and $\hat{\alpha}_{\mathrm{MLE}}$ based on 1000 simulated symmetric stable data sets, each of length $T$ and for tail index $\alpha$. For each box, the center line is the median; the lower and upper edges of the box refer to $q_{25}$ and $q_{75}$ (the 25th and 75th percentiles), respectively; and the whisker length is determined such that they extend to all points not deemed outliers, where an outlier is any point less than $q_{25}-1.5\left(q_{75}-q_{25}\right)$ or greater than $q_{75}+1.5\left(q_{75}-q_{25}\right)$.

This result can be compared to Figure 2, which shows the same plot, but having used Student's $t$ 


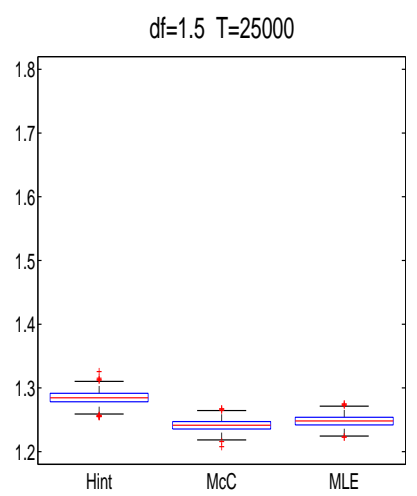

data with $v$ degrees of freedom, $v=1.5,2.5,3.5$ and 4.5, for the sample size $T=25,000$. (The exercise was conducted for various sample sizes and the results are qualitatively exactly the same: we use this rather extreme sample size just to make the point optically very clear.) The discrepancy between $\hat{\alpha}_{\text {Hint }}$ and $\hat{\alpha}_{\mathrm{McC}}$ is readily apparent, and indicates that their asymptotic values converge to constants-and these constants are different when the data are Student's $t$, for $1<v<\infty$ (recall that $v=1$ corresponds to stable with $\alpha=1$, while as $v \rightarrow \infty$, it approaches the normal, or stable with $\alpha=2$ ). Noteworthy is the behavior of the MLE. Not only is $\hat{\alpha}_{\mathrm{McC}}<\hat{\alpha}_{\mathrm{MLE}}<\hat{\alpha}_{\mathrm{Hint}}$ as $T \rightarrow \infty$, but as $v \rightarrow 1$, $\hat{\alpha}_{\mathrm{McC}}$ and $\hat{\alpha}_{\text {MLE }}$ are close, and appear to converge as $T \rightarrow \infty$, while as $v \rightarrow \infty, \hat{\alpha}_{\text {MLE }}$ "moves towards" $\hat{\alpha}_{\text {Hint }}$, and appear to converge. Of course, all three estimators will converge as $v \rightarrow 1$ (to $\alpha=1$ ) and as $v \rightarrow \infty$ (to $\alpha=2$ ), though we see that, for $1<v<\infty$, the behavior of $\hat{\alpha}_{\mathrm{McC}}$ and $\hat{\alpha}_{\mathrm{Hint}}$ always differ.

Figure 2: Similar to Figure 1 but based on simulated Student's $t$ data with df degrees of freedom, and using $T=25,000$. MLE refers to the maximum likelihood estimator of stable tail index $\alpha$.

As such, we consider use of the $\alpha$-Hat Discrepancy Test, or, in short, ALHADI, with test statistic given by

$$
A=A(\mathbf{X})=\hat{\alpha}_{\text {Hint }}(\mathbf{X})-\hat{\alpha}_{\mathrm{McC}}(\mathbf{X}),
$$

for testing the i.i.d. S $\alpha \mathrm{S}$ null hypothesis. Under the null, $\mathbb{E}[A(\mathbf{X})] \approx 0$, recalling that $\hat{\alpha}_{\mathrm{Hint}}$ and $\hat{\alpha}_{\mathrm{McC}}$ are both consistent, with the former being practically unbiased even in small samples. We note that, while it is almost surely the case that there exists some function of the data $\mathbf{X}$ that yields a test with higher power than ALHADI for a range of viable alternatives, it is far from obvious how to find such a statistic. For a specific alternative, the use of likelihood ratio can be used; see Section 3.5 below.

For the Student's $t$ alternative, we saw that $\mathbb{E}[A(\mathbf{X})]>0$. This will not be the case for all alternatives. We consider four further distributions which are suitable for modeling leptokurtic data, and which exhibit different tail behaviors. The first is the two-component Gaussian mixture, such that, for $X \sim \operatorname{MixN}\left(\mu_{1}, \sigma_{1}, \mu_{2}, \sigma_{2}, \lambda\right)$, the pdf of $X$ is $f_{X}(x)=\lambda f_{N}\left(x ; \mu_{1}, \sigma_{1}\right)+(1-\lambda) f_{N}\left(x ; \mu_{2}, \sigma_{2}\right)$, where $0<\lambda<1$ and $f_{N}$ denotes the normal pdf. The use of the MixN for modeling financial asset returns has a substantial history; see Haas et al. (2004), Haas et al. (2013), Paolella (2013), and the references therein. The next is a two-component $\mathrm{S} \alpha \mathrm{S}$ mixture: For $X \sim \operatorname{MixS}\left(\alpha_{1}, \mu_{1}, \sigma_{1}, \alpha_{2}, \mu_{2}, \sigma_{2}, \lambda\right)$ its pdf is $f_{X}(x)=\lambda f_{S}\left(x ; \alpha_{1}, \mu_{1}, \sigma_{1}\right)+(1-\lambda) f_{S}\left(x ; \alpha_{2}, \mu_{2}, \sigma_{2}\right)$, where $0<\lambda<1$ and $f_{S}$ denotes the S $\alpha \mathrm{S}$ pdf. See Broda et al. (2013) and the references therein for the use of stable mixtures for modeling asset returns. 

5 kind with index $\nu$, given by

$$
K_{\nu}(x)=\frac{1}{2} \int_{0}^{\infty} t^{\nu-1} \mathrm{e}^{-\frac{1}{2} x\left(t+t^{-1}\right)} \mathrm{d} t, \quad x>0 .
$$

Clearly, the MixN has short tails, while the MixS has power tails.

The third case we consider is the normal inverse Gaussian, or NIG, distribution, with pdf (allowing for the unfortunate convention of using $\alpha$ and $\beta$ as shape parameters, as in the stable Paretian)

$$
f_{\mathrm{NIG}}(x ; \alpha, \beta, \delta, \mu)=\mathrm{e}^{\delta \sqrt{\alpha^{2}-\beta^{2}}} \frac{\alpha \delta}{\pi \sqrt{\delta^{2}+(x-\mu)^{2}}} K_{1}\left(\alpha \sqrt{\delta^{2}+(x-\mu)^{2}}\right) \mathrm{e}^{\beta(x-\mu)},
$$

for $\alpha>0, \beta \in(-\alpha, \alpha), \delta>0$ and $\mu \in \mathbb{R}$, where $K_{\nu}$ denotes the modified Bessel function of the third

The limiting tail behavior is $f_{\mathrm{NIG}}(x ; \alpha, \beta) \propto|x|^{-3 / 2} \mathrm{e}^{(\mp \alpha+\beta) x}$, as $x \rightarrow \pm \infty$, which is referred to as 'semi-heavy'; see Paolella (2007, Ch. 9) for a detailed presentation of the NIG and its relation to the generalized hyperbolic distribution; and Broda and Paolella (2009) and the references therein for its use in modeling financial returns. As $\alpha \rightarrow 0$, and recalling $0 \leqslant|\beta|<\alpha$, the tail behavior approaches $(2 \pi)^{-1 / 2} x^{-3 / 2}$. This limiting case is known as the Lévy distribution, which coincides with the stable distribution for (now referring to the stable Paretian parameters) $\alpha=1 / 2$ and $\beta=1$.

The fourth case is the generalized asymmetric (Student's) $t$, or GAt, distribution, given in (19) below. Clearly, from Figure 2, for the special case of Student's $t$, the test has power, but we are interested in parameter constellations which best mimic the stable Paretian. To determine such a set of parameters, we take a large simulated sample of $\mathrm{S} \alpha \mathrm{S}$ data with $\alpha=1.5$, and estimate the GAt; this yielded $\hat{d}=2.9073, \hat{\nu}=0.6429, \hat{c}=1.705$, and $\theta=1, \mu=0$. The implied supremum of the maximally existing moment is $\hat{\nu} \hat{d}=1.87$. Simulation of GAt realizations is conducted via the probability integral transform and inverting the cdf.

Figure 3 shows the behavior of the three estimators under these four alternatives, based on $T=$ 25, 000. For the mixed normal, we use $\mu_{1}=\mu_{2}=0, \sigma_{1}=1, \sigma_{2}=4$, and $\lambda=1 / 2$. For the mixed stable, $\mu_{1}=\mu_{2}=0, \sigma_{1}=\sigma_{2}=1, \alpha_{1}=1.2, \alpha_{2}=3-\alpha_{1}=1.8$, and $\lambda=1 / 2$, while for the NIG, $\alpha=0.6$, $\beta=0, \mu=0$ and $\delta=1$. We again see strong separation between $\hat{\alpha}_{\mathrm{Hint}}$ and $\hat{\alpha}_{\mathrm{McC}}$ for the MixN and NIG cases, while the separation in the MixS case depends on the value of $\alpha_{1}$ : As $\alpha_{1}$ moves from 1.5 to 1.0, the separation increases.

For the GAt alternative, it is remarkable that the estimated values of stable tail index $\alpha$ are close to 1.5 (recall how the GAt parameters were selected), and the separation is rather weak. In sample sizes of 5000 or less, the separation is barely apparent. As such, the ALHADI test (9) will have virtually no power against some GAt alternatives. We will see below that only the $\tau_{20}$ and LRT tests have some power, though relatively low, compared to other alternatives, when fixing $d=2.9073$ and varying parameter $\nu$ (the location and scale parameters do not play a role; and we set $\theta$ to its value corresponding to symmetry, i.e., $\theta=1$ ). Thus, the GAt with $d \approx 2.9$ serves as a challenging alternative distribution for future developments of tests for stability.

For the MixS alternative, $\mathbb{E}\left[\hat{\alpha}_{\text {Hint }}(\mathbf{X})\right]<\mathbb{E}\left[\hat{\alpha}_{\mathrm{McC}}(\mathbf{X})\right]$. Our test will return a $p$-value, so that one can reject for either case, and the user can decide between a one- or two-sided test. In our comparisons later, we will always use a one-sided test, rejecting for large values of (9), and not consider the MixS case, though we observe that (9) does have some power against this alternative. 

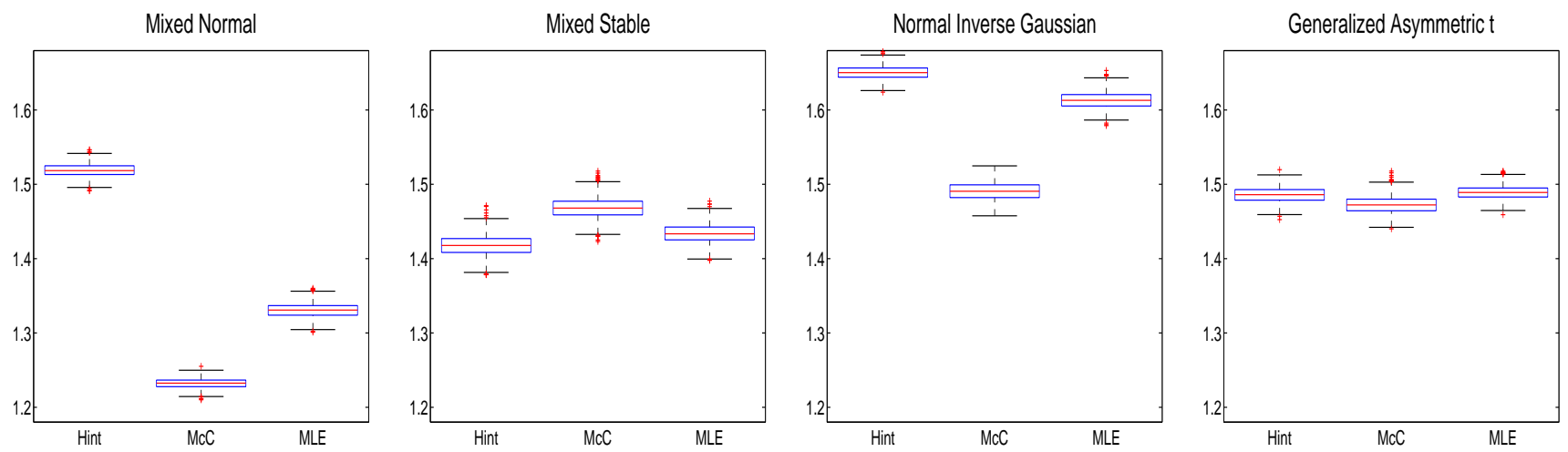

Figure 3: Boxplots of $\hat{\alpha}_{\mathrm{Hint}}, \hat{\alpha}_{\mathrm{McC}}$ and $\hat{\alpha}_{\mathrm{MLE}}$ under four non-stable-Paretian distributional assumptions, based on 1,000 replications, each of length $T=25,000$.

Appendix $\mathrm{C}$ details the method for eliciting $p$-values and illustrates the accuracy of the actual size of the test.

\subsection{Combined Test Procedure}

With the ability to quickly and accurately approximate the $p$-values of the three general tests, they can be combined to form a joint test. We use the same method as discussed in Paolella (2015) in the context of testing composite normality, and join two tests. (The three tests, $D_{T, 5}, \tau_{20}$, and ALHADI, could also easily be combined; but as we will see below, the $D_{T, 5}$ test has rather low power, and does not yield higher power when combined with the other tests.) The constituent tests are not independent, so that the usual $\chi^{2}$ distributional result is not applicable, and simulation is required. We first compute $S_{1}, \ldots, S_{B}$, where the $S_{i}$ are i.i.d., each being the sum of the logs of the two $p$-values under the null of $\mathrm{S} \alpha \mathrm{S}$, for a grid of $\alpha$-values, say $\boldsymbol{\alpha}=(1.05,1.1,1.15, \ldots, 1.95,1.99)$, and $B$ a large number, for which we use 10,000 .

Once these are computed, we can quickly calculate the power of the joint test for a particular data set $\mathbf{X}$ as follows. Having used the sum of logs (and not their negative), for each $\alpha$-value, the fraction of the $S_{i}$ which are less than the sum of the logs of $p$-values corresponding to the constituent tests applied to $\mathbf{X}$ is recorded, and this is stored as a vector, say $\boldsymbol{p}_{C}$. Then, the $p$-value of the combined test, $p_{C}$, is formed via interpolation into the vectors $\boldsymbol{\alpha}$ and $\boldsymbol{p}_{C}$, using $\hat{\alpha}_{\text {Hint }}(\mathbf{X})$. This can be conducted for numerous simulated data sets, and the fraction of the $p_{C}$ values that is less than, say, 0.05 , is the actual size, or power, of the joint test with nominal size 0.05 .

\section{Remarks:}

1. We will see below that the combined test based on $\tau_{20}$ and ALHADI is indeed - and often quite substantially - superior in terms of power compared to its constituent component tests for nearly all distributions and parameter constellations considered.

2. For a single particular data set, given the ability to quickly compute the ALHADI and $\tau_{20} p$ values, the combined test $p$-value is easily computed via simulation based on the actual sample 
size of the data set and its estimate of $\alpha$. The only reason for simulating it over a grid of $\alpha$-values (for a fixed sample size) is so that it can be used for a large number of data sets (of the same length), as required in our size and power simulations, and empirical applications with numerous data sets.

3. The power of the $D_{T, 5}$ test (7) based on the characteristic function is unfortunately rather low for most alternatives of interest. One might be curious to know if, via combining it with the ALHADI test, denoted $A+D$, the resulting power can be enhanced. This turns out to be not the case. The results are illustrated in Section D.

\subsection{Likelihood Ratio Tests}

It is conceivable that there is a composite (i.e., specified distribution but unspecified parameters) alternative in mind to the stable Paretian, in which case, a likelihood ratio test (LRT) could be developed. A very natural candidate is the (location-scale) Student's $t$, being a heavy-tailed distribution with, like the $\mathrm{S} \alpha \mathrm{S}$, one shape parameter, that determines the tail index. In this case, the LRT would not be of the classic form, in which there is a model that nests both alternatives, but rather as a comparison of two non-nested models, along the lines of Vuong (1989). The LRT can exhibit very high power against a specific alternative, and as such, serves to give a feasible upper bound on the power of a test, with respect to that (composite) alternative. Interestingly, we will see below that this LRT also has excellent power properties against other alternatives besides the Student's $t$.

This method can be operationalized for conducting a size 0.05 test of the null of composite $\mathrm{S} \alpha \mathrm{S}$ versus the alternative of composite Student's $t$, for a particular data set $\mathbf{X}$ of length $T$, by performing the following steps:

1. Estimate the parameters of the $\mathrm{S} \alpha \mathrm{S}$ distribution, say $\widehat{\boldsymbol{\theta}}_{0}=(\hat{\alpha}, \hat{\sigma}, \hat{\mu})$, using the MLE, with associated log-likelihood denoted by $\ell_{\mathrm{S} \alpha \mathrm{S}}\left(\widehat{\boldsymbol{\theta}}_{0} ; \mathbf{X}\right)$.

2. Estimate the parameters of the location-scale Student's $t$ distribution and compute the associated $\log$-likelihood $\ell_{t}(\cdot ; \mathbf{X})$.

3. Compute ratio

$$
\operatorname{LR}_{0}(\mathbf{X})=2 \times\left(\ell_{t}(\cdot ; \mathbf{X})-\ell_{\mathrm{S} \alpha \mathrm{S}}\left(\widehat{\boldsymbol{\theta}}_{0} ; \mathbf{X}\right)\right)
$$

4. For $i=1, \ldots, s_{1}$,

(a) Simulate $\mathbf{X}_{(i)}$, consisting of $T$ copies of $\mathrm{S} \alpha \mathrm{S}$ realizations with parameter vector $\widehat{\boldsymbol{\theta}}_{0}$.

(b) Similar to steps 1 to 3 , compute ratio $\operatorname{LR}_{i}\left(\mathbf{X}_{(i)}\right)=2 \times\left(\ell_{t}\left(\cdot ; \mathbf{X}_{(i)}\right)-\ell_{\mathrm{S} \alpha \mathrm{S}}\left(\widehat{\boldsymbol{\theta}}_{i} ; \mathbf{X}_{(i)}\right)\right)$.

5. Reject the $\mathrm{S} \alpha \mathrm{S}$ null hypothesis in favor of the Student's $t$ alternative if $\mathrm{LR}_{0}$ is equal to or exceeds the $95 \%$ empirical quantile of $\left(\mathrm{LR}_{1}, \ldots, \mathrm{LR}_{s_{1}}\right)$. 
However, for the large number of simulations we wish to conduct herein, this parametric bootstrap procedure will be too slow. As such, we proceed similar to the development of the previous tests: For a grid of $\alpha$ values for a fixed $T$ (or a over two-dimensional grid of $T$ and $\alpha$ values), for each element in the grid, simulate (12) $s_{1}$ times, based on $\mathrm{S} \alpha \mathrm{S}$ data, and record the empirical $95 \%$ quantile of the $s_{1}$ LR values. This yields a set of actual cutoff values $c_{\text {act }}(0.05 ; T, \alpha)$. Then, for a particular data set of length $T$, and based on an estimator of $\alpha$, use interpolation into the grid based on the actual $T$ and $\hat{\alpha}$ to approximate the appropriate cutoff value corresponding to a $5 \%$ level test. For three sample sizes $T=500, T=1000$ and $T=2500$, and based on a grid of values $\alpha=1.05,1.1,1.15, \ldots, 1.95,1.99$, the construction of the grid is relatively fast (having using $s_{1}=20,000$ ), assuming fast ML estimation of the $\mathrm{S} \alpha \mathrm{S}$ model, for which we use Nolan's spline approximation to the density, in conjunction with standard Hessian-based general multivariate optimization routines.

Once functions $c_{\text {act }}(0.05 ; T, \alpha)$ are available, the application of the test is very fast. Simulations (based on 10,000 realizations and different seeds than those used to determine the cutoffs) confirm that the test is size-correct to two decimal points for $\mathrm{S} \alpha \mathrm{S}$ data with $1.2 \leqslant \alpha \leqslant 1.9$. For example, for $T=1000$, the average size was 0.0516 (where the true value of $\alpha$ is not used, but rather estimated from the data, as detailed above, and used for interpolating into the grid of simulated cutoff values to get the approximate cutoff value corresponding to a $5 \%$ level test).

To help envision the test, the left panel of Figure 4 shows a kernel density estimate based on a sample of $T=1000$ simulated $\mathrm{S} \alpha \mathrm{S}$ data with $\alpha=1.6$, along with the MLE fitted location-scale $\mathrm{S} \alpha \mathrm{S}$ and Student's $t$ densities. The fitted $\mathrm{S} \alpha \mathrm{S}$ is indeed slightly closer to the kernel density, though the Student's $t$ fit is nearly as good. The right panel shows as a histogram the LRT values, based on $T=1000$, and the (negatives of the) interpolated cutoff values of the test (making them negative just for ease of graphic illustration), based on 1000 replications, using $\mathrm{S} \alpha \mathrm{S}$ data. The cutoff values are all rather close, but not equal because the MLE of $\alpha$ is used for interpolation into the grid of cutoff values. By construction, they cluster around the $95 \%$ quantile of the simulated LRT values.
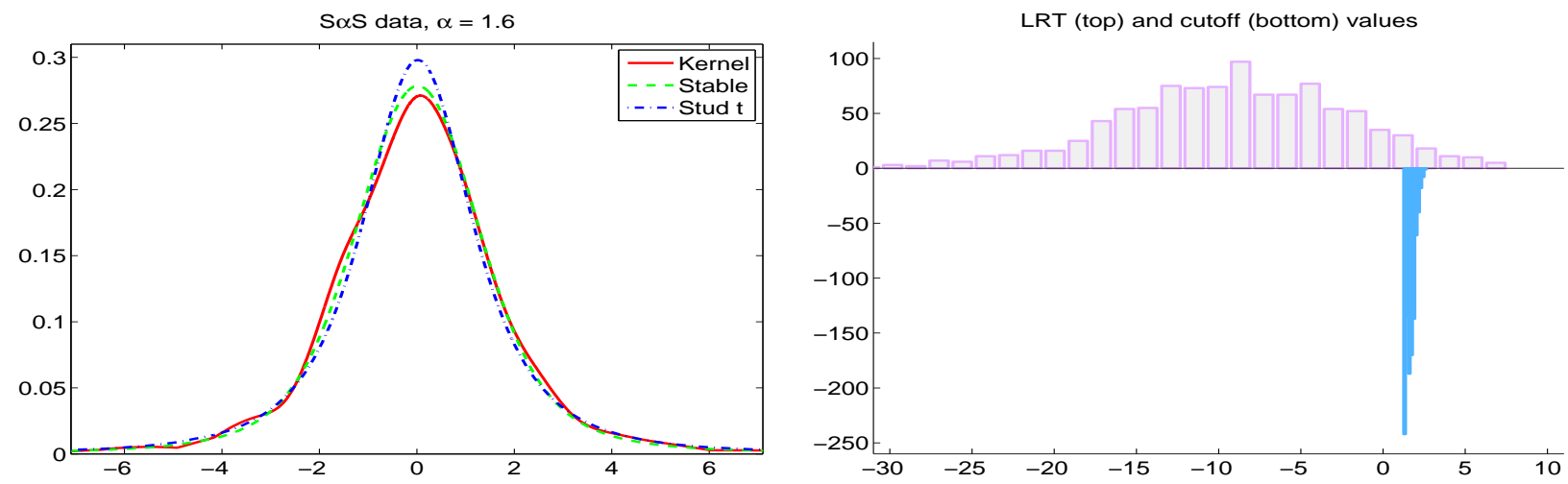

Figure 4: Fitted $\mathrm{S} \alpha \mathrm{S}$ and Student's $t$ to $\mathrm{S} \alpha \mathrm{S}$ data (left) and $1000 \mathrm{LRT}$ and cutoff values (made negative, for graphic illustration) under the null (right). 


\subsection{Actual Size of the Symmetric Stable Tests for Nominal 0.05}

2 For the tests and sample sizes considered in the subsequent section on power, we first illustrate the 3 actual sizes obtained, for several values of stable tail index $\alpha$. Table 1 contains the results. All results 4 are based on $s=10,000$ replications, and such that, in each column, the same $s$ data sets were used.

5 The last row of the table shows the average of the $\hat{\alpha}_{\text {Hint }}$ estimates over the $s$ samples. The most accurate 6 tests with respect to a nominal size of $5 \%$ are $A+\tau_{20}$ and LRT - and conveniently so, as these will be 7 seen to have the highest power. Further reporting on the actual sizes can be found in the Appendix.

\begin{tabular}{|c|c|c|c|c|c|c|c|c|c|c|c|c|c|c|c|c|}
\hline \multicolumn{2}{|c|}{ Sample size } & \multicolumn{5}{|c|}{$T=500$} & \multicolumn{5}{|c|}{$T=1000$} & \multicolumn{5}{|c|}{$T=2500$} \\
\hline Test & Eq. & 1.3 & 1.45 & 1.60 & 1.75 & 1.90 & 1.3 & 1.45 & 1.60 & 1.75 & 1.90 & 1.3 & 1.45 & 1.60 & 1.75 & 1.90 \\
\hline$D_{T, 5}$ & (7) & 0.068 & 0.053 & 0.050 & 0.053 & 0.050 & 0.050 & 0.048 & 0.050 & 0.050 & 0.046 & 0.045 & 0.046 & 0.050 & 0.051 & 0.051 \\
\hline$\tau_{20}$ & (8) & 0.069 & 0.060 & 0.055 & 0.045 & 0.035 & 0.056 & 0.056 & 0.051 & 0.049 & 0.038 & 0.053 & 0.050 & 0.051 & 0.050 & 0.036 \\
\hline$A$ & (9) & 0.058 & 0.045 & 0.042 & 0.046 & 0.055 & 0.050 & 0.046 & 0.043 & 0.046 & 0.055 & 0.049 & 0.045 & 0.046 & 0.048 & 0.055 \\
\hline$A+\tau_{20}$ & & 0.051 & 0.052 & 0.051 & 0.050 & 0.054 & 0.050 & 0.052 & 0.051 & 0.050 & 0.050 & 0.050 & 0.050 & 0.050 & 0.051 & 0.050 \\
\hline LRT & $(12)$ & 0.052 & 0.050 & 0.049 & 0.053 & 0.051 & 0.051 & 0.050 & 0.050 & 0.049 & 0.053 & 0.049 & 0.048 & 0.048 & 0.052 & 0.056 \\
\hline Average & $\hat{\alpha}_{\text {Hint }}$ & 1.30 & 1.45 & 1.60 & 1.75 & 1.90 & 1.30 & 1.45 & 1.60 & 1.75 & 1.90 & 1.30 & 1.45 & 1.60 & 1.75 & 1.90 \\
\hline
\end{tabular}

Table 1: Actual sizes of tests, for i.i.d. symmetric stable data with tail index $\alpha=1.3,1.45,1.6,1.75,1.9$, for sample size $T$, using the nominal size of $5 \%$.

\subsection{Power}

For assessing the power of the four general (non-specific alternative) tests discussed herein for the symmetric case, and the LRT, we will use the Student's $t$ and the various alternatives discussed in Figure 3 above. We restrict ourselves to (i) varying only one of the parameters of the alternative distribution; (ii) use of the nominal size of $5 \%$, as it the most common in practice; and (iii) use of three sample sizes, $T=500, T=1000$ and $T=2500$. All reported powers are based on use of $s=10,000$ replications, and such that, in each column of the forthcoming tables, the same $s$ data sets were used.

Beginning with the Student's $t$ alternative, Table 2 shows the power of the four general-alternative tests, and the LRT, for six values of the degrees of freedom parameter, $v=1.5,2,3,4,5,6$. The last row of the table shows the average of the $\hat{\alpha}_{\text {Hint }}$ estimates over the $s=10,000$ replications. For all tests, the power decreases as $v \rightarrow 1$ and $v \rightarrow \infty$ as expected. In all cases, the $D_{T, 5}$ test has the lowest power, and demonstrably so. Using the notation $a>b$ to indicate that test $a$ has higher power than $b$, and $a \vee b=\max (\operatorname{power}(a)$, power $(b))$, we see that, for $v=1.5$ and $v=2, A>\tau_{20}$, while for $v \geqslant 3, \tau_{20}>A$. However, in both cases, we have $A+\tau_{20}>\left(A \vee \tau_{20}\right)$ (except at $v=1.5$, in which case the powers of $A+\tau_{20}$ and $A$ differ at most by 0.01 , but most likely in this case, this is due to $A$ having slightly too liberal size; see Table 1), with the power of the combined test often substantially higher than $A \vee \tau_{20}$.

For all three sample sizes and for all $v$, the LRT dominates, as must be the case asymptotically for the Student's $t$ alternative, by construction. Interestingly, for $v=4$, the powers of $A+\tau_{20}$ and LRT are rather close ( 0.52 and 0.57 , respectively, for $T=500$; and 0.82 and 0.88 for $T=1000$ ). This is relevant, because $v=4$ is considered to be the most typical, and best "default choice" degrees of freedom value for financial asset returns data; see Platen and Heath (2006, p. 90). 


\begin{tabular}{|c|c|c|c|c|c|c|c|c|c|c|c|c|c|c|c|c|c|c|c|}
\hline \multicolumn{2}{|c|}{ Sample size } & \multicolumn{6}{|c|}{$T=500$} & \multicolumn{6}{|c|}{$T=1000$} & \multicolumn{6}{|c|}{$T=2500$} \\
\hline Test & Eq. & 1.5 & 2 & 3 & 4 & 5 & 6 & 1.5 & 2 & 3 & 4 & 5 & 6 & 1.5 & 2 & 3 & 4 & 5 & 6 \\
\hline$D_{T, 5}$ & (7) & 0.12 & 0.10 & 0.10 & 0.11 & 0.10 & 0.10 & 0.09 & 0.12 & 0.14 & 0.13 & 0.12 & 0.11 & 0.09 & 0.16 & 0.29 & 0.28 & 0.23 & 0.20 \\
\hline$\tau_{20}$ & (8) & 0.18 & 0.29 & 0.42 & 0.42 & 0.35 & 0.29 & 0.22 & 0.46 & 0.72 & 0.74 & 0.68 & 0.59 & 0.28 & 0.65 & 0.95 & 0.98 & 0.98 & 0.97 \\
\hline$A$ & (9) & 0.18 & 0.30 & 0.36 & 0.32 & 0.27 & 0.23 & 0.30 & 0.54 & 0.61 & 0.54 & 0.44 & 0.38 & 0.59 & 0.90 & 0.94 & 0.88 & 0.79 & 0.69 \\
\hline$A+\tau_{2}$ & & 0.17 & 0.36 & 0.54 & 0.52 & 0.45 & 0.37 & 0.29 & 0.61 & 0.82 & 0.82 & 0.74 & 0.66 & 0.59 & 0.93 & 0.99 & 0.99 & 0.99 & 0.97 \\
\hline LRT & $(12)$ & 0.27 & 0.46 & 0.61 & 0.57 & 0.53 & 0.50 & 0.44 & 0.71 & 0.89 & 0.88 & 0.85 & 0.81 & 0.77 & 0.97 & 1.00 & 1.00 & 1.00 & 0.99 \\
\hline Avera & $\hat{\alpha}_{\text {Hint }}$ & 1.28 & 1.46 & 1.64 & 1.73 & 1.79 & 1.82 & 1.28 & 1.46 & 1.64 & 1.73 & 1.79 & 1.82 & 1.29 & 1.45 & 1.64 & 1.73 & 1.79 & 1.82 \\
\hline
\end{tabular}

Table 2: Power against the Student's $t$ alternative, for degrees of freedom values $v=1.5,2,3,4,5,6$ and sample size $T$, using the nominal size of $5 \%$.

We can now confirm our conjecture that the $\tau_{20}$ test should outperform the original $\tau$ test proposed in Paolella (2001). For example, for $T=2500$ and $v=4$, the power of $\tau_{20}$ is 0.98 , while for the original $\tau$, it was only 0.50 . That the performance would be so much better was not anticipated.

Table 3 shows the power for a range of MixN alternatives at the $5 \%$ nominal testing level. Having chosen this alternative for investigation was fortuitous, as we have a case (the only one) for which the power of $D_{T, 5}$ test is strong, and such that, for a segment of the parameter space (depending on sample size $T$ ), $D_{T, 5}>\tau_{20}$. Observe that the power of $\tau_{20}$ begins to decrease as a function of $\sigma_{2}$ (with the starting point depending on the sample size). It decreases so much that, at some point, the test is biased for level $5 \%$, i.e., the power is less than the size under the alternative. Closer inspection indicates that the $\tau_{20}$ test still can have some value for this alternative: When conducted at the $10 \%$ level, the powers for $T=2500$ and $\sigma_{2}=2,3,4,5,6$ are 1.0, 1.0, 0.96, 0.76, and 0.54, respectively. Nevertheless, as with the Student's $t$ case, $A+\tau_{20}>\left(A \vee \tau_{20}\right)$ and $A+\tau_{20}>D_{T, 5}$.

\begin{tabular}{|c|c|c|c|c|c|c|c|c|c|c|c|c|c|c|c|c|}
\hline \multicolumn{2}{|c|}{ Sample size } & \multicolumn{5}{|c|}{$T=500$} & \multicolumn{5}{|c|}{$T=1000$} & \multicolumn{5}{|c|}{$T=2500$} \\
\hline Test & Eq. & 2 & 3 & 4 & 5 & 6 & 2 & 3 & 4 & 5 & 6 & 2 & 3 & 4 & 5 & 6 \\
\hline$D_{T, 5}$ & (7) & 0.14 & 0.41 & 0.74 & 0.90 & 0.93 & 0.20 & 0.81 & 0.98 & 1.00 & 1.00 & 0.51 & 1.00 & 1.00 & 1.00 & 1.00 \\
\hline$\tau_{20}$ & (8) & 0.52 & 0.84 & 0.67 & 0.52 & 0.41 & 0.87 & 0.95 & 0.72 & 0.46 & 0.32 & 0.99 & 0.77 & 0.09 & 0.01 & 0.01 \\
\hline$A$ & (9) & 0.49 & 0.98 & 1.00 & 1.00 & 1.00 & 0.75 & 1.00 & 1.00 & 1.00 & 1.00 & 0.98 & 1.00 & 1.00 & 1.00 & 1.00 \\
\hline$A+\tau_{2}$ & & 0.71 & 1.00 & 1.00 & 1.00 & 1.00 & 0.95 & 1.00 & 1.00 & 1.00 & 1.00 & 1.00 & 1.00 & 1.00 & 1.00 & 1.00 \\
\hline LRT & (12) & 0.90 & 0.97 & 1.00 & 0.99 & 0.79 & 0.99 & 0.97 & 1.00 & 1.00 & 0.87 & 0.99 & 0.99 & 1.00 & 1.00 & 0.96 \\
\hline Avera & $\hat{\alpha}_{\text {Hint }}$ & 1.80 & 1.61 & 1.52 & 1.48 & 1.46 & 1.80 & 1.61 & 1.52 & 1.48 & 1.46 & 1.80 & 1.61 & 1.52 & 1.48 & 1.46 \\
\hline Averag & $\hat{\alpha}_{\mathrm{MLE}}$ & 1.85 & 1.55 & 1.34 & 1.19 & 1.08 & 1.85 & 1.55 & 1.34 & 1.19 & 1.08 & 1.85 & 1.55 & 1.33 & 1.19 & 1.08 \\
\hline
\end{tabular}

Table 3: Power against the MixN alternative, with pdf $f_{X}(x)=(1 / 2) f_{N}(x ; 0,1)+(1 / 2) f_{N}\left(x ; 0, \sigma_{2}\right)$, for 2 nd component scale values $\sigma_{2}=2,3,4,5,6$, using the nominal size of $5 \%$.

What is perhaps unexpected is the high power of the LRT test, given that it has been designed for a specific (composite) alternative of Student's $t$. To help envision the test in this case, Figure 5 is similar to Figure 4, but having used simulated MixN data with $\sigma_{2}=3$ (and again based on $T=1000$ ). The fitted $\mathrm{S} \alpha \mathrm{S}$ and Student's $t$ distributions are very close, and both differ significantly from the MixN density, so that one might have expected the LRT test statistic to be close to one, and the test to have low power. Yet as revealed by the right panel, this is not the case. This occurs because the Student's $t$ can accommodate the mixed normal shape slightly (but enough for a test) better than the stable. As seen in Table 3, except for the $\sigma_{2}=6$ case, it has power nearly equivalent to $A+\tau_{20}$. The reason for 


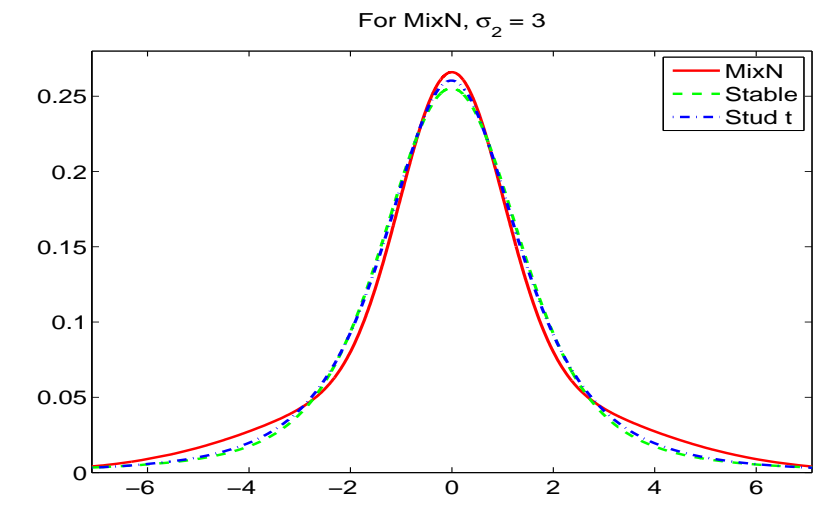
be applicable as $\sigma_{2}$ grows. the breakdown as $\sigma_{2}$ increases is that the MLE of $\alpha$ tends towards one as $\sigma_{2}$ increases, as seen in the last line of the table, but the LRT was only calibrated for $1.05 \leqslant \alpha \leqslant 1.99$. As such, the test will not

Figure 5: Fitted $\mathrm{S} \alpha \mathrm{S}$ and Student's $t$ to MixN data (left) and 1000 LRT and cutoff values under the alternative of MixN (right).

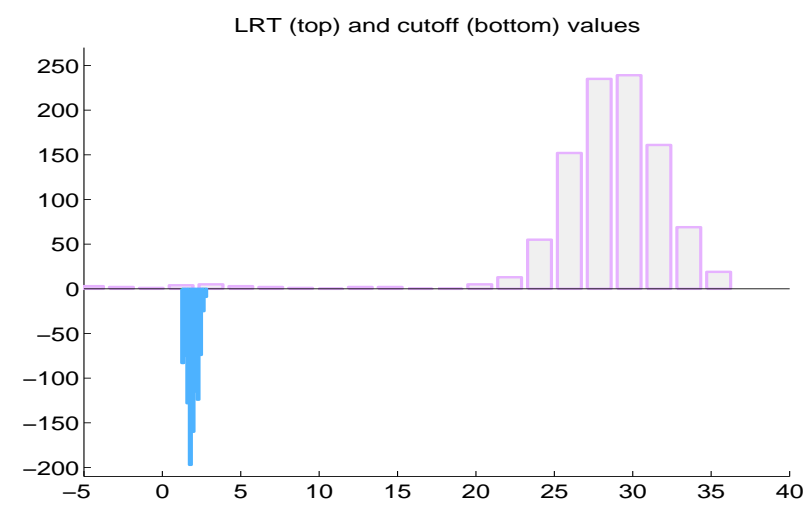

Next, consider the NIG alternative, with pdf (10). Table 4 is similar to the previous two tables, showing the power as the NIG shape parameter $\alpha$ varies. As expected, for all tests, the power decreases as NIG shape parameter $\alpha$ increases. As in the Student's $t$ case, the $D_{T, 5}$ test performs relatively poorly. For the other three non-specific tests, we have a uniformity result across all used sample sizes and values of NIG shape parameter: $A+\tau_{20}>\tau_{20}>A$, with the combined test $A+\tau_{20}$ having substantially higher power than $\tau_{20}$ for the smaller sample sizes. Between $A+\tau_{20}$ and the LRT, the results are not uniform, neither with respect to shape parameter $\alpha$, nor with respect to sample size, except that power increases for both as the sample size increases, as must be the case for consistent tests.

\begin{tabular}{|c|c|c|c|c|c|c|c|c|c|c|c|c|c|c|c|c|}
\hline \multicolumn{2}{|c|}{ Sample size } & \multicolumn{5}{|c|}{$T=500$} & \multicolumn{5}{|c|}{$T=1000$} & \multicolumn{5}{|c|}{$T=2500$} \\
\hline Test & Eq. & 0.3 & 0.6 & 0.9 & 1.2 & 1.5 & 0.3 & 0.6 & 0.9 & 1.2 & 1.5 & 0.3 & 0.6 & 0.9 & 1.2 & 1.5 \\
\hline$D_{T, 5}$ & (7) & 0.20 & 0.19 & 0.16 & 0.14 & 0.12 & 0.36 & 0.33 & 0.26 & 0.20 & 0.17 & 0.85 & 0.82 & 0.68 & 0.52 & 0.41 \\
\hline$\tau_{20}$ & (8) & 0.83 & 0.75 & 0.65 & 0.55 & 0.45 & 0.99 & 0.98 & 0.95 & 0.88 & 0.80 & 1.00 & 1.00 & 1.00 & 1.00 & 1.00 \\
\hline$A$ & (9) & 0.78 & 0.69 & 0.57 & 0.46 & 0.40 & 0.98 & 0.93 & 0.84 & 0.74 & 0.63 & 1.00 & 1.00 & 0.99 & 0.98 & 0.94 \\
\hline$A+\tau_{20}$ & & 0.94 & 0.89 & 0.81 & 0.69 & 0.61 & 1.00 & 1.00 & 0.98 & 0.94 & 0.88 & 1.00 & 1.00 & 1.00 & 1.00 & 1.00 \\
\hline LRT & $(12)$ & 0.98 & 0.75 & 0.87 & 0.85 & 0.80 & 1.00 & 0.87 & 0.96 & 0.95 & 0.95 & 1.00 & 0.97 & 0.99 & 0.98 & 0.97 \\
\hline Average & Iint & 1.53 & 1.65 & 1.72 & 1.76 & 1.79 & 1.53 & 1.65 & 1.72 & 1.76 & 1.79 & 1.53 & 1.65 & 1.72 & 1.76 & 1.79 \\
\hline
\end{tabular}

Table 4: Power against the NIG alternative, with pdf (10), using $\beta=0, \mu=0, \delta=1$, and shape values $\alpha=$ $0.3,0.6,0.9,1.2,1.5$, for the nominal size of $5 \%$.

Recall the GAt distribution (19) which, when calibrated to stable Paretian data, renders the ALHADI test nearly powerless, as shown in Figure 3. We consider this case, varying parameter $v$, with power shown in Table 5 . Indeed, the ALHADI and $D_{T, 5}$ tests perform poorly, and such that the tests are biased, with some power values lower than the size. The $\tau_{20}$ test performs the best overall, dominating all other tests in most cases. The $\tau_{20}$ and LRT tests have their maximal power at (for the values considered) $\nu=1$, which corresponds to a maximally existing moment of $\nu d \approx 2.9$. As $\theta=1$ (symmetric case), the GAt reduces to the Cauchy distribution for $\nu=1 / d \approx 0.34$, and converges to an 
exponential-tail distribution as $\nu \rightarrow \infty$; this explains the decrease in power as $\nu$ decreases or increases away from 1.0. Unlike in the Student's $t$, NIG and mixed normal cases, the LRT test is not the most powerful, but performs well overall. As $\nu$ decreases, the MLE of stable parameter $\alpha$ decreases, and the LRT test will not be applicable, for the same reason as mentioned for the MixN case. This explains why the LRT is biased and has nearly zero power for $\nu=0.4$.

\begin{tabular}{|c|c|c|c|c|c|c|c|c|c|c|c|c|c|c|c|c|}
\hline \multicolumn{2}{|c|}{ Sample size } & \multicolumn{5}{|c|}{$T=500$} & \multicolumn{5}{|c|}{$T=1000$} & \multicolumn{5}{|c|}{$T=2500$} \\
\hline Test & Eq. & 0.4 & 0.7 & 1.0 & 1.3 & 1.6 & 0.4 & 0.7 & 1.0 & 1.3 & 1.6 & 0.4 & 0.7 & 1.0 & 1.3 & 1.6 \\
\hline$D_{T, 5}$ & (7) & 0.13 & 0.06 & 0.07 & 0.05 & 0.05 & 0.10 & 0.06 & 0.06 & 0.05 & 0.04 & 0.04 & 0.06 & 0.07 & 0.06 & 0.04 \\
\hline$\tau_{20}$ & (8) & 0.07 & 0.21 & 0.23 & 0.15 & 0.08 & 0.07 & 0.33 & 0.40 & 0.26 & 0.14 & 0.07 & 0.52 & 0.79 & 0.70 & 0.41 \\
\hline$A$ & (9) & 0.09 & 0.07 & 0.05 & 0.03 & 0.02 & 0.07 & 0.09 & 0.06 & 0.02 & 0.01 & 0.03 & 0.13 & 0.06 & 0.01 & 0.00 \\
\hline$A+\tau_{2}$ & & 0.06 & 0.16 & 0.15 & 0.09 & 0.04 & 0.04 & 0.24 & 0.26 & 0.12 & 0.05 & 0.03 & 0.42 & 0.54 & 0.34 & 0.10 \\
\hline LRT & (12) & 0.02 & 0.15 & 0.20 & 0.11 & 0.05 & 0.01 & 0.21 & 0.36 & 0.22 & 0.15 & 0.00 & 0.43 & 0.73 & 0.61 & 0.64 \\
\hline Averag & $\hat{\mathbf{x}}_{\text {Hint }}$ & 1.14 & 1.54 & 1.73 & 1.84 & 1.90 & 1.14 & 1.54 & 1.73 & 1.84 & 1.90 & 1.14 & 1.54 & 1.73 & 1.84 & 1.90 \\
\hline
\end{tabular}

Table 5: Power against the GAt alternative, with pdf (19), using $d=2.9073$ and $\theta=1$ (and $\mu=0, c=1.7$, though the location and scale terms are irrelevant for power considerations), and shape values $\nu=0.4,0.7,1.0,1.3,1.6$, for nominal size of $5 \%$.

The last alternative we consider is (a particular form of) the MixS distribution, with pdf $f_{X}(x)=$ $(1 / 2) f_{S}\left(x ; \alpha_{1}, 0,1\right)+(1 / 2) f_{S}\left(x ; 3-\alpha_{1}, 0,1\right)$, where $f_{S}$ denotes the $\mathrm{S} \alpha \mathrm{S}$ pdf. The tabulated power values are not shown. Use of a two-sided ALHADI test would have power, but based on the observation in Figure 3, the power will be very low for this alternative. The $\tau_{20}$ test is not expected to have power against this alternative, as sums of the data do not submit to the Gaussian central limit theorem. Indeed, this is the case: except for $\alpha_{1}=1.5$, which reduces to $\mathrm{S} \alpha \mathrm{S}$ with tail index 1.5, the power of $\tau_{20}$ for $\alpha_{1}>1.5$ is below the nominal size. This is also the case with the $D_{T, 5}$ test.

Remark: Recall that, in the MixN case, the LRT has high power because the Student's $t$ fits the MixN adequately better than the $\mathrm{S} \alpha \mathrm{S}$. One might conjecture that the stable might fit better than the Student's $t$ and the LRT test would have power in its left tail for distributions which are "closer" to the stable, such as the MixS, as well as the tempered stable (see Küchler and Tappe, 2013 and the references therein) and the geometric stable (see, e.g., Kozubowski and Rachev, 1999, Kozubowski, 2000, Halvarsson, 2013, and the numerous references therein).

This conjecture is confirmed in Figure 6, which is similar to Figures 4 and 5, but for MixS data with $\alpha_{1}=1.7$ (and again based on $T=1000$ ). (For this data generating process, the average estimated stable tail index over the 1000 simulations was 1.47 , which is very close to the average of 1.7 and 1.3. This average being well over 1.2, it also confirms that the LRT test can be computed in this case.) Comparing the right panels of Figures 5 and 6 , it is clear that the LRT will have power against the MixS alternative, but the power is nowhere near as strong as the LRT against the MixN, as seen from the right panel of Figure 5 .

Based on this observation, we would be behooved to use a two-sided LRT test, and protocol the $2.5 \%$ and $97.5 \%$ quantiles instead of only the $95 \%$ quantile, if such "stable like" alternatives are of interest. The MixS case we use here being somewhat of a toy example, we leave the development of testing procedures of stable versus the more interesting and relevant cases of tempered and geometric 
stable for future research.
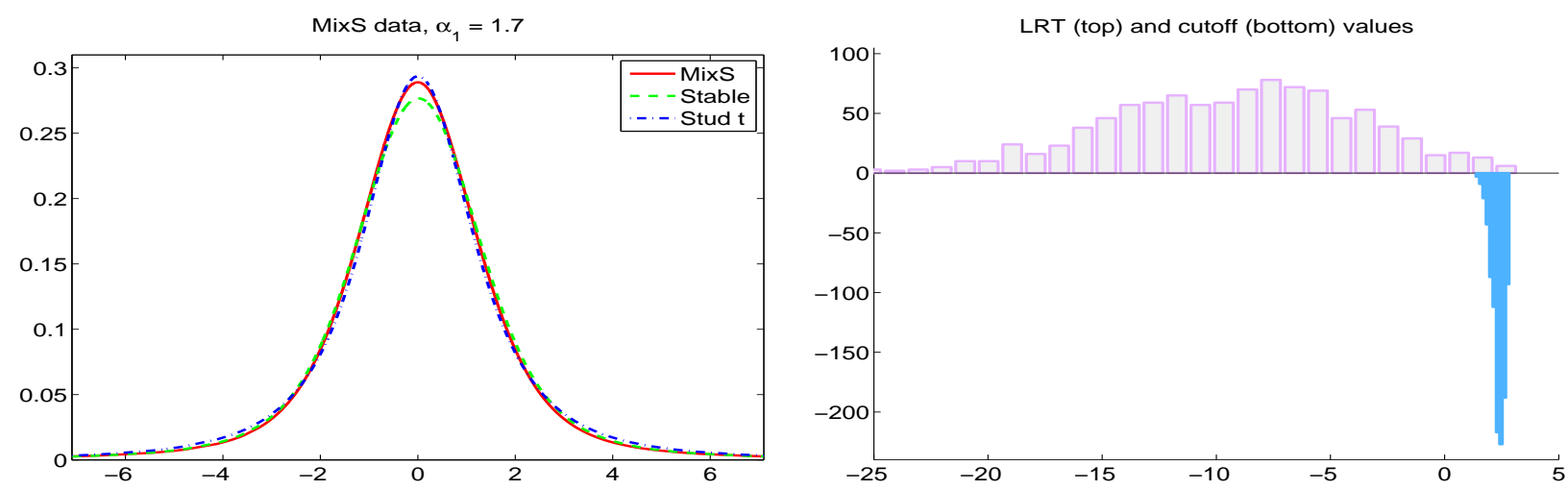

Figure 6: Fitted S $\alpha$ S and Student's $t$ to MixS data (left) and 1000 LRT and cutoff values under the alternative of MixS (right).

\section{Extension to Testing the Asymmetric Stable Paretian Case}

\section{$3 \quad 4.1 \quad$ Ignoring the Asymmetry}

4 Let $X_{t} \sim S_{\alpha, \beta}(\mu, \sigma)$ be i.i.d. copies, $t=1, \ldots, T$, each with $\operatorname{cdf} F_{X}(\cdot ; \alpha, \beta, \mu, \sigma)$. First consider what 5 happens to the actual size of the $\tau_{20}$ and ALHADI tests when asymmetry is ignored. This is con6 ducted via simulation using 10,000 replications, based on the nominal size of 5\%, for the single (and 7 reasonably typical for financial asset returns) value of $\alpha=1.6$, three sample sizes, and values of $8 \beta=0, \pm 0.2, \pm 0.4, \pm 0.6, \pm 0.8$.

The results are shown in Table 6. Both tests appear to have actual size symmetric in $\beta$, and such that they become too liberal as $|\beta| \rightarrow 1$, much more so for ALHADI. The actual size of the ALHADI test not only breaks down as $|\beta|$ moves away from zero, but gets worse as the sample size increases; whereas the size of the $\tau_{20}$ test is still somewhat reasonable across sample sizes and for moderate $\beta$, and its actual size even improves (mildly) for positive $\beta$ as the sample size grows, but worsens for negative $\beta$. Thus, for data sets that only exhibit a mild amount of asymmetry, the $\tau_{20}$ test can still be used, though this conclusion is limited, as we studied only the $\alpha=1.6$ case. As generation of stable random variates and computation of the $\tau_{20}$ test are very fast, one can always use simulation, based on the MLE of $\alpha$ and $\beta$, for a particular data set, to assess the actual size for a given nominal size.

\subsection{Use of CDF and Inverse CDF Transform}

Our simple idea to deal with the asymmetric case is to apply the aforementioned tests for S $\alpha \mathrm{S}$ data to $\tilde{\mathbf{X}}=\left(\tilde{X}_{1}, \ldots, \tilde{X}_{T}\right)$, where

$$
\tilde{X}_{t}=F_{X}^{-1}\left(P_{t} ; \hat{\alpha}_{\mathrm{MLE}}, 0,0,1\right), \quad P_{t}=F_{X}\left(\left(X-\hat{\mu}_{\mathrm{MLE}}\right) / \hat{\sigma}_{\mathrm{MLE}} ; \hat{\alpha}_{\mathrm{MLE}}, \hat{\beta}_{\mathrm{MLE}}, 0,1\right),
$$

$t=1, \ldots, T$, i.e., the data are transformed by the inverse cdf based on the MLE to produce $\mathrm{S} \alpha \mathrm{S}$ data. This procedure is numerically feasible using the fast MLE, cdf, and inverse cdf routines provided in 


\begin{tabular}{lllllllllll}
\hline & & \multicolumn{10}{c}{$T=500$} \\
\cline { 3 - 10 } Test & Eq. & 0.8 & 0.6 & 0.4 & 0.2 & 0 & -0.2 & -0.4 & -0.6 & -0.8 \\
\cline { 3 - 11 }$\tau_{20}$ & $(8)$ & 0.14 & 0.10 & 0.075 & 0.061 & 0.066 & 0.070 & 0.083 & 0.098 & 0.14 \\
$A$ & $(9)$ & 0.39 & 0.23 & 0.12 & 0.047 & 0.040 & 0.067 & 0.13 & 0.24 & 0.40 \\
& & & & & & & & & & \\
& & & & & $T=1,000$ & & & & \\
$\tau_{20}$ & $(8)$ & 0.13 & 0.093 & 0.069 & 0.057 & 0.051 & 0.057 & 0.072 & 0.094 & 0.13 \\
$A$ & $(9)$ & 0.55 & 0.32 & 0.15 & 0.065 & 0.043 & 0.068 & 0.15 & 0.32 & 0.55 \\
& & & & & & & & & & \\
$\tau_{20}$ & $(8)$ & 0.12 & 0.082 & 0.059 & 0.048 & 0.047 & 0.061 & 0.082 & 0.11 & 0.18 \\
$A$ & $(9)$ & 0.82 & 0.49 & 0.20 & 0.073 & 0.049 & 0.087 & 0.23 & 0.51 & 0.83 \\
\hline
\end{tabular}

Table 6: Actual sizes of the $\tau_{20}$ and ALHADI nominal 5\% tests, as designed for symmetric stable data, but applied to asymmetric stable data, using 10,000 replications, and based on $\alpha=1.6$ and $\beta=0, \pm 0.2, \pm 0.4, \pm 0.6, \pm 0.8$, using sample size $T$, and ignoring the asymmetry.

Nolan's toolbox, and clearly asymptotically valid, though in finite samples, and in light of the relative difficulty of estimating $\beta$ accurately, can result in size distortions, depending on the nature of the test.

Table 7 is similar to Table 6, but having applied transform (13), and also showing the results for the combined test $A+\tau_{20}$ and LRT. As conjectured, the actual sizes of all the tests improve as the sample size increases, and also, for a given sample size, decrease away from 0.05 as $|\beta|$ increases. The actual sizes of $\tau_{20}$ and the LRT are quite reasonable for $|\beta| \leqslant 0.6$, as is that of the combined test for $|\beta| \leqslant 0.4$.

We turn now to the power of these tests for two asymmetric alternatives. We use the asymmetric NIG distribution (10), with $\alpha=1.2$ and $\beta=0,-0.3,-0.6,-0.9$; and the noncentral Student's $t$ with $v=4$ degrees of freedom and noncentrality (asymmetry) parameters $\gamma=0,-0.6,-1.2,-1.8$, these values having been chosen to capture mild, strong and very strong asymmetry, relative to financial returns data. Recall the (singly) noncentral $t$, hereafter NCT, distribution is given as follows. Let $Z \sim \mathrm{N}\left(\gamma, \sigma^{2}\right)$, and $Y \sim \chi^{2}(v)$, independently of $Z$. Then

$$
X=\mu+\frac{Z}{\sqrt{Y / v}} \sim \operatorname{NCT}(\mu, \sigma ; v, \gamma)
$$

where $\mu$ and $\gamma$ are location and noncentrality coefficients, respectively, $v \in \mathbb{R}_{>0}$ denotes the degrees of freedom parameter, $\sigma$ is the scale parameter, and

$$
\mathbb{E}[X]=\mu+\zeta, \text { if } v>1, \quad \zeta=\gamma(v / 2)^{1 / 2} \Gamma((v-1) / 2) / \Gamma(v / 2) .
$$

(A detailed discussion of the singly and doubly noncentral $t$ distributions, including closed-form vectorized saddlepoint approximations to the density, is provided in Paolella, 2007, Ch. 10.)

Table 8 shows the results based on 1000 replications. We see that, for the NCT and NIG alternatives, the power of all the tests weakens as the asymmetry grows in magnitude. We have, for all three distributional alternatives considered, the ordering with respect to power among the first four tests as LRT $>A+\tau_{20}>\tau_{20}>A$. The fifth test will be discussed below. 


\begin{tabular}{lcccccccccc}
\hline & & \multicolumn{10}{c}{$T=500$} \\
\cline { 3 - 10 } Test & Eq. & 0.8 & 0.6 & 0.4 & 0.2 & 0 & -0.2 & -0.4 & -0.6 & -0.8 \\
\cline { 3 - 11 }$\tau_{20}$ & $(8),(13)$ & 0.034 & 0.043 & 0.050 & 0.051 & 0.052 & 0.051 & 0.049 & 0.045 & 0.032 \\
$A$ & $(9),(13)$ & 0.029 & 0.031 & 0.034 & 0.035 & 0.035 & 0.034 & 0.035 & 0.031 & 0.032 \\
$A+\tau_{20}$ & $(13)$ & 0.028 & 0.035 & 0.042 & 0.042 & 0.044 & 0.043 & 0.042 & 0.036 & 0.029 \\
LRT & $(12),(13)$ & 0.031 & 0.042 & 0.050 & 0.052 & 0.052 & 0.052 & 0.050 & 0.043 & 0.031 \\
& & & & & & & & & & \\
$\tau_{20}$ & $(8),(13)$ & 0.034 & 0.048 & 0.050 & 0.052 & 0.050 & 0.053 & 0.052 & 0.049 & 0.034 \\
$A$ & $(9),(13)$ & 0.034 & 0.038 & 0.038 & 0.039 & 0.039 & 0.037 & 0.038 & 0.039 & 0.032 \\
$A+\tau_{20}$ & $(13)$ & 0.031 & 0.043 & 0.046 & 0.047 & 0.047 & 0.047 & 0.046 & 0.043 & 0.029 \\
LRT & $(12),(13)$ & 0.031 & 0.045 & 0.050 & 0.051 & 0.052 & 0.052 & 0.050 & 0.044 & 0.029 \\
& & & & & & & & & & \\
$\tau_{20}$ & $(8),(13)$ & 0.034 & 0.049 & 0.050 & 0.051 & 0.051 & 0.050 & 0.049 & 0.046 & 0.031 \\
$A$ & $(9),(13)$ & 0.029 & 0.040 & 0.042 & 0.043 & 0.042 & 0.044 & 0.042 & 0.042 & 0.030 \\
$A+\tau_{20}$ & $(13)$ & 0.029 & 0.044 & 0.048 & 0.049 & 0.048 & 0.047 & 0.047 & 0.047 & 0.030 \\
LRT & $(12),(13)$ & 0.029 & 0.045 & 0.051 & 0.051 & 0.052 & 0.052 & 0.052 & 0.048 & 0.030 \\
\hline
\end{tabular}

Table 7: Similar to Table 6, showing actual size for a nominal size of 5\%, again based on sample size $T$ and using 10,000 replications, but accounting for asymmetry by having applied transform (13). Also shown are the actual sizes of the combined test $A+\tau_{20}$ and LRT.

\begin{tabular}{|c|c|c|c|c|c|c|c|c|c|c|}
\hline \multicolumn{2}{|c|}{ Alternative } & \multicolumn{4}{|c|}{ Noncentral $t$} & \multicolumn{4}{|c|}{ Asymmetric NIG } & \multirow[t]{2}{*}{ IHS } \\
\hline Test & Eq. & 0.0 & -0.6 & -1.2 & -1.8 & 0.0 & -0.3 & -0.6 & -0.9 & \\
\hline$\tau_{20}$ & $(8),(13)$ & 0.71 & 0.70 & 0.40 & 0.13 & 0.89 & 0.81 & 0.63 & 0.40 & 0.91 \\
\hline$A$ & $(9),(13)$ & 0.50 & 0.42 & 0.20 & 0.11 & 0.72 & 0.65 & 0.43 & 0.27 & 0.75 \\
\hline$A+\tau_{20}$ & (13) & 0.79 & 0.73 & 0.42 & 0.17 & 0.94 & 0.90 & 0.69 & 0.44 & 0.95 \\
\hline LRT & $(12),(13)$ & 0.90 & 0.84 & 0.44 & 0.17 & 0.99 & 0.94 & 0.78 & 0.51 & 0.99 \\
\hline LRT & (17) & 0.92 & 0.92 & 0.85 & 0.29 & 1.00 & 0.98 & 0.88 & 0.28 & 0.99 \\
\hline
\end{tabular}

Table 8: For $T=1000$ and nominal size 5\%, powers against asymmetric alternatives of the $\tau_{20}$, ALHADI, combined $A+\tau_{20}$, and LRT (12) tests, having used transform (13); and, in the last row, LRT (17). The left panels show the power for the noncentral Student's $t$ (14), based on $v=4$ degrees of freedom and noncentrality (asymmetry) parameters $\gamma=0,-0.6,-1.2,-1.8$. The center panels use the asymmetric NIG (10) with NIG shape parameters $\alpha=1.2$ and $\beta=0,-0.3,-0.6,-0.9$. The rightmost column is for the IHS distribution (16) with $\lambda=-0.17$ and $\theta=0.82$. 


\section{Remarks:}

1. One might conjecture that the reason for the poor performance of the LRT for the GAt alternative, and good performance of the LRT for NIG and MixN, is that both (noncentral) Student's $t$ and NIG are continuous location scale mixtures of normals (see, e.g., Paolella, 2007, p. 267, and Ch. 9 for detailed discussion), and the MixN is a discrete mixture of normals. If so, then the LRT would be expected to have relatively high power against numerous alternatives, most notably the generalized hyperbolic and its other special cases besides the NIG. To substantiate this conjecture, we inspect its power (and that of the other tests) against another leptokurtic, asymmetric alternative that is not a normal mixture; namely the inverse hyperbolic sine (IHS) distribution, attributed to Johnson (1949) and used for empirical financial applications by Brooks et al. (2005) and, most notably, Choi and Nam (2008).

Random variable $Y$ follows an IHS distribution, denoted $Y \sim \operatorname{IHS}(\lambda, \theta)$, if $\sinh ^{-1}(Y) \sim \mathrm{N}\left(\lambda, \theta^{2}\right)$ or, with $Z \sim \mathrm{N}(0,1), Y=\sinh (\lambda+\theta Z)$. As $\sinh ^{-1}(\cdot)$ is a nondecreasing function of its argument, the pdf of $Y$ is straightforwardly obtained and given by

$$
f_{Y}(y ; \lambda, \theta)=\frac{1}{\sqrt{2 \pi\left(y^{2}+1\right) \theta^{2}}} \exp \left\{-\frac{\left(\sinh ^{-1}(y)-\lambda\right)^{2}}{2 \theta^{2}}\right\} .
$$

The IHS distribution is very convenient for financial applications, as the VaR can be explicitly determined. In particular, using

$$
w=\frac{1}{\theta}\left(\sinh ^{-1}(x)-\lambda\right)=\frac{1}{\theta}\left(\ln \left(x+\sqrt{1+x^{2}}\right)-\lambda\right), \quad \mathrm{d} w=\frac{\mathrm{d} x}{\theta \sqrt{1+x^{2}}}
$$

yields

$$
F_{Y}(y ; \lambda, \theta)=\int_{-\infty}^{\frac{1}{\theta}\left(\ln \left(y+\sqrt{1+y^{2}}\right)-\lambda\right)} f_{Z}(w) \mathrm{d} w=\Phi\left(\frac{1}{\theta}\left(\sinh ^{-1}(y)-\lambda\right)\right),
$$

where $\Phi$ is the standard normal cdf, and the quantile function is

$$
\operatorname{Pr}\left(Y \leqslant y_{q}\right)=\operatorname{Pr}\left(\sinh (\lambda+\theta Z) \leqslant y_{q}\right) \Rightarrow F_{Y}^{-1}(q)=\sinh \left(\lambda+\theta \Phi^{-1}(q)\right) .
$$

For a power comparison, we consider only one parameter set, obtained by fitting the locationscale IHS to a large sample of simulated $S_{1.7,-0.3}(0,1)$ data, these being typical values for financial returns data, yielding IHS shape parameters $\lambda=-0.17$ and $\theta=0.82$. Figure 7 shows the four parameter stable and four parameter fitted IHS distribution (along with a kernel density estimate of simulated values from the latter, confirming the method of simulation).

The rightmost column of Table 8 shows the power of the various tests against the IHS distribution. In this case, the LRT dominates with a power of 0.99 , followed by the combined test $A+\tau_{20}$, with power 0.95. Thus, it appears that the LRT can have the best power for (a segment of the parameter space of) distributions that are not location-scale continuous or discrete mixtures of normals, thus refuting our simple conjecture and adding further support to the use of the LRT against a variety of alternatives. 


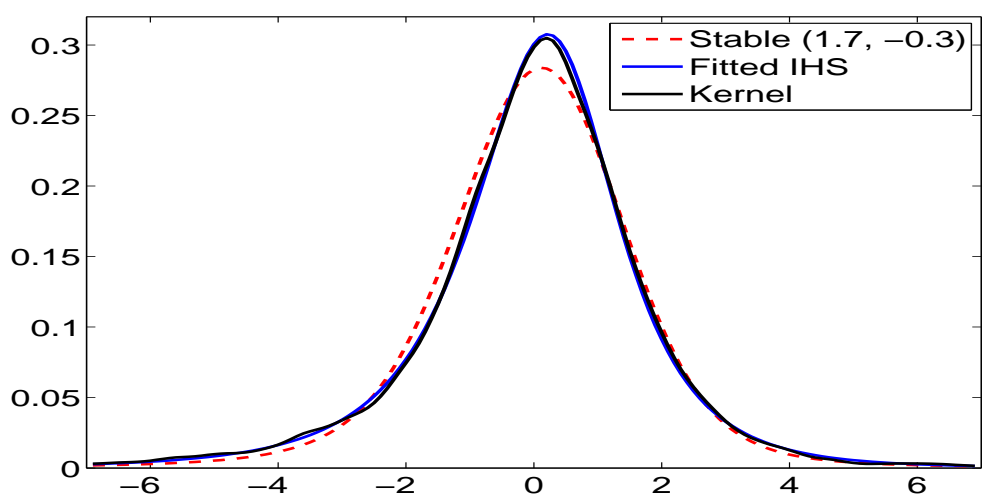

Figure 7: The $S_{1.7,-0.3}(0,1)$ density (dashed) and the best-fitting location-scale IHS density (yielding $\lambda=-0.17$ and $\theta=0.82)$, based on the MLE from a sample of size $T=50,000$ of $S_{1.7,-0.3}(0,1)$ data; and a kernel density estimate of simulated IHS realizations based on the four parameters of the fitted distribution.

2. In light of the excellent performance of the LRT for several alternatives, one naturally considers the development of combined (ideally two-sided, recalling the LRTs performance with the MixS) tests via the method in Section 3.4 using LRTs for a variety of judiciously chosen alternatives. This could lead to a very powerful test for sensible alternatives to the stable Paretian distribution for parameter constellations typical in finance and other applications. We leave such ideas for future research.

\subsection{LRT for Asymmetric Stable versus Noncentral Student's $t$}

The composite LRT can be extended to the asymmetric stable case. This requires applying the procedure discussed in Section 3.5 to a two-dimensional grid of $\alpha$ and $\beta$ values for a fixed sample size, for which we use $T=1000$ (or a three-dimensional grid also using various sample sizes), and forming the likelihood ratio based on the likelihood of the fitted location-scale asymmetric stable and a particular asymmetric fat-tailed alternative. For this, we use the NCT (14), generalizing the Student's $t$ as we used in the symmetric LRT case, so that

$$
\operatorname{LR}_{0}(\mathbf{X})=2 \times\left(\ell_{\mathrm{NCT}}(\cdot ; \mathbf{X})-\ell_{S_{\alpha, \beta}}\left(\widehat{\boldsymbol{\theta}}_{0} ; \mathbf{X}\right)\right)
$$

where now $\widehat{\boldsymbol{\theta}}_{0}=(\hat{\alpha}, \hat{\beta}, \hat{\sigma}, \hat{\mu})$. We chose the NCT instead of other asymmetric generalizations of the Student's $t$, such as the GAt with $d=2$, or the interesting constructions by Lye and Martin (1993) and Jones and Faddy (2003), because the NCT possesses the desirable property of being closed under addition, in the sense that sums of margins of the Kshirsagar (1961) multivariate NCT is again NCT (see, e.g., Kotz and Nadarajah, 2004), so that, for example, if a set of asset returns is modeled using a multivariate NCT, then the portfolio distribution is analytically tractable; see, e.g., Jondeau (2010). The idea of using the (univariate) NCT distribution for modeling asset returns goes back to Harvey and Siddique (1999).

For the stable MLE, we use the canned method in Nolan's toolbox, based on a spline approximation to the stable density, this being quite fast and accurate enough for our purposes; see below for further 
discussion. The NCT density at a given point, as an infinite sum or integral, is relatively slow to evaluate, and computing the MLE requires doing this for $T=1000$ data points, and this many times during an optimization process. To form the cutoff values, this needs to be computed for a grid of $\alpha$ and $\beta$ values, each (say) 1000 times; in particular, we require the MLE for $20 \times 37 \times 1000=740,000$ data sets. As such, the standard MLE approach cannot be used. Fortunately, there is a better way. The four parameters of the location-scale NCT can be estimated extraordinarily fast by computing

$$
\arg \max _{\mu, \sigma} f_{\mathrm{NCT}^{*}}(\mathbf{X} ; \tilde{v}, \tilde{\gamma}, \mu, \sigma), \quad \tilde{v}, \tilde{\gamma}=\mathrm{KP}(\mathbf{Y}), \quad \mathbf{Y}=\frac{\mathbf{X}-\mu}{\sigma},
$$

where $\operatorname{NCT}^{*}(v, \gamma)=\operatorname{NCT}(-\zeta, 1 ; v, \gamma), \zeta$ is given in (15), and $\mathrm{KP}$ refers to the nearly instantaneous (and as accurate as MLE) method of estimation of the two shape parameters based on empirical quantiles and table lookup for the $\mathrm{NCT}^{*}$ distribution, as developed and detailed in Krause and Paolella (2014). Starting values are taken to be $v=4, \gamma=0$, the $50 \%$ trimmed mean for $\mu$ and (based on (15) with $v=4$ and $\gamma=0)\left(s^{2} / 2\right)^{1 / 2}$ for $\sigma$, where $s^{2}$ denotes the sample variance. The likelihood is still required in (18), and this can be evaluated using the fast vectorized closed-form saddlepoint approximation in Broda and Paolella (2007) or the fast density approximation given in Krause and Paolella (2014).

The cutoff values for $T=1000$ corresponding to (17) and a nominal size of $5 \%$ were computed for each element in the two-dimensional grid based on $\alpha=1.05,1.1,1.15, \ldots, 1.95,1.99$, and $\beta=-0.9$, $-0.85,-0.8, \ldots, 0.85,0.9$, as discussed in Section 3.5, based on 1000 replications. The actual size of this procedure, when using estimates of $\alpha$ and $\beta$ and bivariate interpolation into the grid of stored cutoff values, is quite reasonable for $1.5 \leqslant \alpha \leqslant 1.9$ and zero to moderate asymmetry of either sign, as shown in Figure 8 (the average of the plotted points is 0.0504).

However, as the asymmetry decreases towards -1 and increases towards 1 , the actual size becomes more variable and is in almost all cases too low. (The inaccuracy outside this range arises because of the limitations of the spline density approximation to the stable pdf. Some greater accuracy could be achieved for larger $|\beta|$ in the cutoff table by using the FFT approach for computing the density, when the former reports potential problems, though this will incur quite some numeric cost. For developing a cutoff grid with accuracy for $0.6<|\beta| \leqslant 1$ and $1<\alpha<1.4$, yet slower methods of computing the density would be required, and the computing times would become ominous.) As such, application of the test against the NCT, NIG, and IHS for the parameters used in Table 8, and to financial asset returns data, are valid, though as the asymmetry increases, the power will be artificially somewhat lower, given the lower actual size.

The resulting powers, listed in the last line of Table 8, indicate that the method is overall superior to use of the symmetric LRT (12) with transformation (13). The powers are greater for the NCT, as should be expected, with a near doubling of the power for the $\gamma=-1.2$ case. For the NIG, the asymmetric LRT delivers much lower power for the extreme asymmetry case of NIG parameter $\beta=-0.9$, though with the previous comments about the actual size in mind, the reported power in this case is not accurate, and would be higher if the cutoff values for high asymmetry were determined with greater accuracy. 


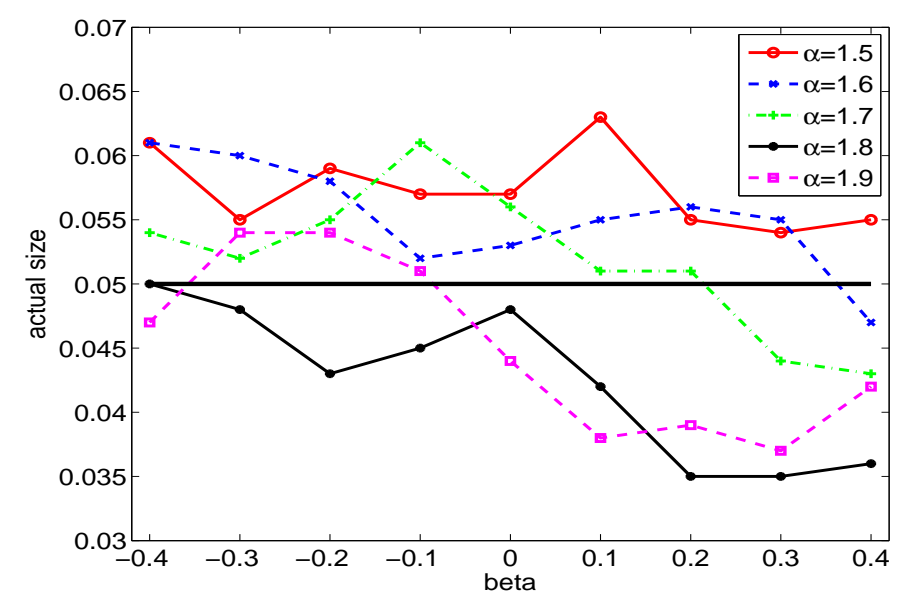

Figure 8: Actual size of the nominal 5\% level LRT (17), for sample size $T=1000$, using a ratio of noncentral Student's $t$ and asymmetric stable Paretian.

\section{Conclusions and Concurrent Research}

2 The fact that the accurate determination of the maximally existing moment of a set of data is very 3 difficult renders application of such methods for assessing the existence of second moments virtually 4 useless in deciding to accept or dismiss the stable assumption. In light of this, and the fact that 5 one can no longer argue that - irrespective of its suitability - the stable distribution is computationally 6 prohibitive, we develop three general-alternative testing procedures, and two likelihood ratio tests for composite alternatives, all designed for $1<\alpha<2$ (though realistically applicable for about $1.3<\alpha<$ 1.95 based on actual size performance) and for both the symmetric and asymmetric stable cases, the latter realistically for about $|\beta|<0.6$.

For the stated ranges, these tests exhibit good actual size properties and, among the general tests, the combined $A+\tau_{20}$ test delivers admirable power against competing alternatives such as Student's $t$, mixed normal, and NIG, and far greater power than the test based on the empirical characteristic function. The two LRTs both perform the best against the alternatives they were designed for, but also very well - often best - against a range of other alternatives.

We emphasize that, without a test with unit power against all alternatives, lack of rejection of the null of stability does not prove that the data are stable. Indeed, other distributional assumptions such as the (asymmetric) Student's $t$ (with heavy tails but such that the tail index lies in $\mathbb{R}_{+}$instead of $(0,2))$, NIG (with semi-heavy tails) and the mixed normal (with short tails) can all be useful models depending on the application - such as risk prediction or portfolio optimization. On the other hand, even rejection of the stable hypothesis does not imply that it is "completely inappropriate" to use it as the error distribution in a model that exhibits heavy tails, asymmetry, and, crucially, for which there is not enough data to fully characterize the tail. Nevertheless, rejection of the stable hypothesis can be a valuable signal to indicate that other heavy-tailed, asymmetric distributions might be more appropriate for the data in question.

With respect to modeling of financial asset returns data, particularly daily and higher frequency, the 
near-ubiquitous stylized fact of volatility clustering renders an i.i.d. setting of little use for short-term risk and density forecasting. In this context, GARCH-type models have established themselves as a viable way of dealing with a time-varying scale term, though the filtered returns - these being much closer to i.i.d.- still exhibit leptokurtic and asymmetric behavior. As such, interest then would center on testing if these residuals come from a stable Paretian distribution. To operationalize this, it is best to fit a GARCH model with stable Paretian innovations, termed an $S_{\alpha, \beta}$-GARCH process (as opposed to use of a two-step procedure based on quasi-ML), and then test if the filtered (location and time-varyingscale-standardized) returns follow a stable distribution. This is pursued in Paolella (2016), in which very fast, non-likelihood-based methods of estimation are proposed, this being relevant particularly when testing large numbers of series is of interest, and then the tests in this paper - with their fast calculation of $p$-values - can be applied. Observe that the validity of such an application depends on the extent to which the filtered innovation sequence (i) is close enough to being i.i.d., noting that any imposed GARCH-type process is surely wrong w.p.1, and (ii) its resulting distribution is "close" to that of the actual innovation sequence. The findings there, conducted on numerous equity return series, suggest that there is strong evidence against the stable as the correct innovations assumption for all stocks and time periods, though for many stocks and windows of data, the stable hypothesis cannot be rejected.

\section{A Determination of $p$-Values for $D_{T, \kappa}(\mathbf{X})$ Test $(7)$}

The goal is to model not just the two or three usual cutoff quantiles of the distribution under the null, but rather the entire distribution, so that $p$-values can be elicited. This is conducted by fitting a flexible distribution to values of the distribution obtained by simulation, for a variety of sample size $T$ and tail index values $\alpha$, and storing the fitted parameters. For an actual data set to be tested, the $p$-value is then computed for each stored distribution in the table (this being fast, because the distribution is chosen such that, in addition to being flexible, its cdf is quickly evaluated), and then interpolation is used based on the actual sample size and estimate of $\alpha$.

For the test based on the empirical and theoretical characteristic function, we proceed as follows.

(i) Simulate $n=10,000$ realizations of (7) under the null, for each element in a grid of sample sizes $T$ and tail indexes $\alpha$, for which we use $T=250,500,750,1000,1500,2000, \ldots, 10,000$; and $\alpha=1.05,1.1,1.2, \ldots, 1.9,1.99$. We use the McCulloch (1986) estimator (being consistent and instantly computed) to obtain $\hat{\mu}$ and $\hat{\sigma}$, as required for the location-scale transformation.

(ii) Fit a flexible parametric distribution (such that the cdf is easily evaluated) to the (log of) the $n$ values for each $T / \alpha$ combination in step 1, and store the estimated parameters.

For (ii), we use a two-component mixture of generalized asymmetric $t$ distribution, or MixGAt. The pdf of the (single component) GAt is given by

$$
f_{\mathrm{GA} t}(z ; d, \nu, \theta)=K \times\left[\left(1+\frac{(-z \theta)^{d}}{\nu}\right)^{-\left(\nu+\frac{1}{d}\right)} \mathbb{I}(z<0)+\left(1+\frac{(z / \theta)^{d}}{\nu}\right)^{-\left(\nu+\frac{1}{d}\right)} \mathbb{I}(z \geqslant 0)\right]
$$


where $d, \nu, \theta \in \mathbb{R}_{>0}$ and $K^{-1}=\left(\theta^{-1}+\theta\right) d^{-1} \nu^{1 / d} B(1 / d, \nu)$, with $B(\cdot, \cdot)$ the beta function. It is noteworthy because limiting cases include the generalized exponential (GED), and hence the Laplace and normal, while the Student's $t$ (and, thus, the Cauchy) distributions are special cases. For $\theta>1(\theta<1)$ the distribution is skewed to the right (left), while for $\theta=1$, it is symmetric. See Paolella (2007, p. $273)$ for further details. The cdf of $Z \sim \operatorname{GA} t(d, \nu, \theta)$ is

$$
F_{Z}(z ; d, \nu, \theta)=\frac{\bar{B}_{L}(\nu, 1 / d)}{1+\theta^{2}} \mathbb{I}(z \leqslant 0)+\frac{\bar{B}_{U}(1 / d, \nu)}{1+\theta^{-2}}+\left(1+\theta^{2}\right)^{-1} \mathbb{I}(z>0),
$$

where $\bar{B}$ is the incomplete beta ratio (or regularized incomplete beta function) given by $\bar{B}_{x}(a, b)=$ $B_{x}(a, b) / B(a, b)$ for $0 \leqslant x \leqslant 1 ; L=\nu /\left(\nu+(-z \theta)^{d}\right)$, and $U=(z / \theta)^{d} /\left(\nu+(z / \theta)^{d}\right)$. As the cdf admits a closed-form expression, the $p$-value corresponding to a particular $\tau_{B}$ test statistic can be quickly computed.

With five parameters (including location and scale), the GAt is a rather flexible distribution. However, it was found to fit the simulated data not as well as desired. Greater accuracy can be obtained by using a two-component mixture of GAt, with mixing parameters $0<\lambda_{1}<1$ and $\lambda_{2}=1-\lambda_{1}$. This 11 parameter construction is extraordinarily flexible, and was found to fit the simulated test statistics essentially perfectly, as confirmed from kernel density plots. Its pdf and cdf are just weighted sums of GAt pdfs and cdfs respectively, so that evaluation of the cdf is no more involved than that of the GAt.

Observe that steps (i) and (ii) of this simulation exercise need to be conducted only once. Then, for a particular data set $\mathbf{X}$ of interest:

(iii) Compute $\mathbf{Y}=\left(Y_{1}, \ldots, Y_{T}\right)$, where $Y_{j}=\left(X_{j}-\hat{\mu}\right) / \hat{\sigma}$, and $\hat{\mu}$ and $\hat{\sigma}$ are based on the McCulloch estimator.

(iv) Compute statistic $D_{T, \kappa}(\mathbf{X})$ from (7), using $\hat{\alpha}_{\text {Hint }}$ as the estimator for the tail index, given its higher accuracy than the McCulloch estimator and its low computational cost.

(v) Build a matrix of $p$-values, say $\mathbf{P}_{D}$, based on the parametric MixGAt approximations, for each entry in the $T / \alpha$ grid, where each $p$-value is the right tail area from $D_{T, \kappa}(\mathbf{X})$ (recall we wish to reject for large values of $(7))$ of the fitted MixGA $t$ distribution.

(vi) Use bivariate interpolation (as implemented in Matlab's interp2 procedure) to deliver the $p$-value, based on the actual length $T$ and estimated tail index $\hat{\alpha}_{\text {Hint }}$ of the data set of interest to index into table $\mathbf{P}_{D}$. (Of course, to enhance speed, if $T$ coincides with one of the entries used in the grid, only that row of the grid is computed, and univariate interpolation is used.)

The actual sizes of the test, for the usual three nominal sizes of $10 \%, 5 \%$ and $1 \%$, were checked, first using the true value of $\alpha$ instead of estimator $\hat{\alpha}_{\text {Hint }}$, and sample sizes which were used in the computation of the grid (and using a different set of seed values for the generation of the stable data sets), so that interpolation of $p$-values was not required. As expected, the actual sizes were very close to their nominal counterparts, confirming that the method of construction of the $p$-value table, via use of the parametric approximation MixGAt, is effective. 
Of course, of real interest are the actual sizes when an estimator is used for $\alpha$, in which case interpolation into the matrix of $p$-values is required. We find that the results are not satisfactory for small sample sizes (e.g., $T=250$ ) and values of $\alpha<1.6$. The reason is that the tail cutoff values corresponding to the three sizes change nonlinearly as a function of $\alpha$, so that, even though the distribution of $\hat{\alpha}_{\text {Hint }}$ is symmetric about the true $\alpha$, the actual size will not agree with the nominal size. This problem can be addressed by using a much denser grid of $\alpha$-values; however, such a scheme would entail an enormous amount of simulation. We propose a second way.

Let $P_{T, \alpha}(\hat{\alpha})$ denote the random variable associated with the $p$-value of $\mathrm{S} \alpha \mathrm{S}$ data $\mathbf{X}=\left(X_{1}, \ldots, X_{T}\right)$ with tail index $\alpha$, based on the procedure given by steps (iii) to (vi) above, using estimator $\hat{\alpha}=$ $\hat{\alpha}_{\text {Hint }} \in[1,2]$ to index into the $p$-value table. Then, for a fixed $T$ and nominal cutoff probability $c_{\text {nom }}$, $c_{\text {nom }} \in\{0.10,0.05,0.01\}$, the actual size $c_{\text {act }}$ as a function of $c_{\text {nom }}$ is

$$
c_{\text {act }}\left(c_{\text {nom }}\right)=\operatorname{Pr}\left(P_{T, \alpha}(\hat{\alpha}) \leqslant c_{\text {nom }}\right)=\mathbb{E}\left[\mathbb{I}\left\{P_{T, \alpha}(\hat{\alpha}) \leqslant c_{\text {nom }}\right\}\right]=\int_{1}^{2} \mathbb{I}\left\{P_{T, \alpha}(a) \leqslant c_{\text {nom }}\right\} f_{\hat{\alpha}}(a) \mathrm{d} a,
$$

where $\mathbb{I}\{A\}$ is the indicator function of event $A$ and $f_{\hat{\alpha}}$ denotes the density of $\hat{\alpha}_{\text {Hint }}$ As an example, imagine that, for a fixed $T$ and true $\alpha=1.55, \hat{\alpha}_{\text {Hint }}$ only takes on values in $(1.45,1.64)$, and that nearestneighbor interpolation is used for determining the $p$-value from the matrix of computed $p$-values $\mathbf{P}_{D}$. Thus, given the symmetry of $f_{\hat{\alpha}}$, only the tabulated $p$-values corresponding to $\alpha=1.5$ and $\alpha=1.6$ are used, with equal probability for a given data set, so that the resulting $p$-value is, on average, the average of those two values. The nonlinearity of the tabulated $p$-values as a function of $\alpha$ is what causes $c_{\text {act }}\left(c_{\text {nom }}\right) \neq c_{\text {nom }}$. The argument of course still holds for $\hat{\alpha}_{\text {Hint }} \in[1,2]$ and use of other methods of interpolation, such as linear or cubic spline.

To resolve this, observe that the mean value theorem for integrals implies that there exists a constant $\alpha_{k}=\alpha_{k}\left(T, \alpha, c_{\text {nom }}\right) \in[1,2]$ such that the integral in (21) equals

$$
\mathbb{I}\left\{P_{T, \alpha}\left(\alpha_{k}\right) \leqslant c_{\mathrm{nom}}\right\} \int_{1}^{2} f_{\hat{\alpha}}(a) \mathrm{d} a=\mathbb{I}\left\{P_{T, \alpha}\left(\alpha_{k}\right) \leqslant c_{\mathrm{nom}}\right\},
$$

i.e., $\exists \alpha_{k}$ such that $c_{\text {act }}=\operatorname{Pr}\left(P_{T, \alpha}\left(\alpha_{k}\right) \leqslant c_{\text {nom }}\right)=c_{\text {nom }}$.

To operationalize this, we let $m=m\left(T, \alpha, c_{\text {nom }}\right)=\alpha_{k}\left(T, \alpha, c_{\text {nom }}\right) / \hat{\alpha}_{\text {Hint }}$, and find via simulation the values of $m$ for a small set of $T$ and a grid of $\alpha$-values. For interpolation into the vector of $p$-values, we use linear interpolation (as opposed to nearest neighbor or cubic spline), because it has the effect of making the values of $m$ closest to unity. The obtained values, based on 10,000 replications, are plotted in Figure 9. We see that almost all are below 1.0, very much so for $\alpha<1.6$, confirming the nonlinearity of the $p$-values as $\alpha$ changes. As expected, as the sample size moves from $T=250$ to 1000 , the values of $m$ are all closer to unity.

Having determined this mapping, for a given data set $\mathbf{X}$, linear interpolation into the vector of

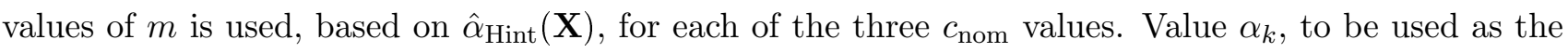
value of $\alpha$ to index the table of $p$-values, is taken to be

$$
\alpha_{k}=\alpha_{k}\left(\mathbf{X}, c_{\mathrm{nom}}\right)=m\left(T, \hat{\alpha}_{\mathrm{Hint}}(\mathbf{X}), c_{\mathrm{nom}}\right) \times \hat{\alpha}_{\mathrm{Hint}}(\mathbf{X}),
$$

for each of the three values of $c_{\text {nom. }}$. Observe that, once the values of $m$ are computed, this method entails no more computational time than the original test and the interpolation to get the $p$-value. We 

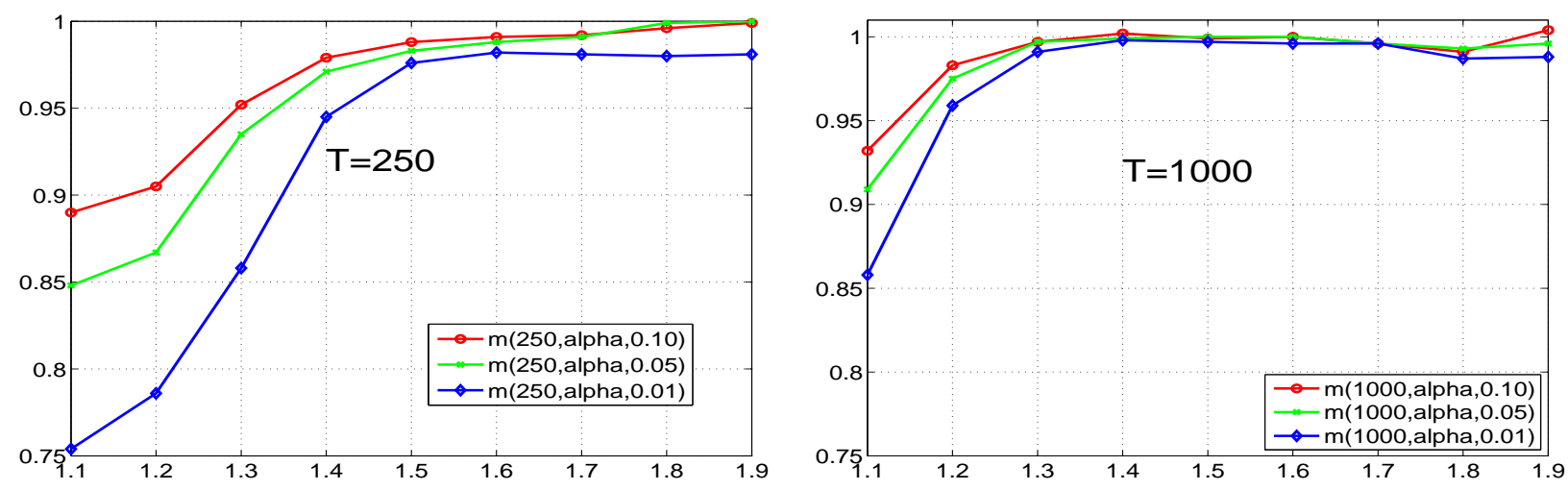

Figure 9: Values of $m\left(T, \alpha, c_{\text {nom }}\right)$, as a function of stable tail index $\alpha$, based on 10,000 replications, for the $D_{T, \kappa}(\mathbf{X})$ test $(7)$ for the two sample sizes $T=250$ (left) and $T=1000$ (right).

expect the use of this method to assist for values $1<\alpha<1.6$; while, given that the true $m$ is near unity for $1.6<\alpha<2$, and the fact that the estimator of $\alpha$ is stochastic (in this case, $\hat{\alpha}_{\text {Hint }}$ ), performance could worsen as $\alpha$ moves towards two.

This is precisely the case, as demonstrated in Table 9, which shows the actual levels corresponding to levels of $5 \%$ and 1\% (similar conclusions hold for the less interesting case of $10 \%$ and are not shown), using the above developed procedure for $T=250$ and $T=1000$ (shown in italics) and otherwise, using the standard method, without augmentation by factor $m$. The results become very good as the sample size $T$ increases, because $\hat{\alpha}_{\text {Hint }}$ is consistent for $\alpha$ and the use of the MixGAt approximation (for any sample size and $\alpha$ ) is very accurate.

\begin{tabular}{|c|c|c|c|c|c|c|c|c|c|c|}
\hline \multirow{2}{*}{$\begin{array}{l}\text { Nominal } \\
T \backslash \alpha\end{array}$} & \multicolumn{5}{|c|}{0.05} & \multicolumn{5}{|c|}{0.01} \\
\hline & 1.20 & 1.35 & 1.55 & 1.75 & 1.90 & 1.20 & 1.35 & 1.55 & 1.75 & 1.90 \\
\hline 250 & 0.086 & 0.062 & 0.049 & 0.043 & 0.017 & 0.0373 & 0.0184 & 0.0096 & 0.0070 & 0.0024 \\
\hline 250 & 0.129 & 0.093 & 0.060 & 0.052 & 0.050 & 0.0643 & 0.0290 & 0.0136 & 0.0136 & 0.0143 \\
\hline 1000 & 0.059 & 0.048 & 0.047 & 0.050 & 0.022 & 0.0181 & 0.0082 & 0.0092 & 0.0096 & 0.0030 \\
\hline 1000 & 0.075 & 0.050 & 0.048 & 0.054 & 0.051 & 0.0267 & 0.0095 & 0.0096 & 0.0120 & 0.0109 \\
\hline 2500 & 0.050 & 0.043 & 0.049 & 0.051 & 0.051 & 0.0103 & 0.0075 & 0.0076 & 0.0108 & 0.0114 \\
\hline 5000 & 0.045 & 0.042 & 0.049 & 0.052 & 0.052 & 0.0087 & 0.0065 & 0.0074 & 0.0103 & 0.0112 \\
\hline
\end{tabular}

Table 9: Actual sizes of the nominal $5 \%$ and $1 \% D_{T, \kappa}(\mathbf{X})$ test $(7)$ for data of length $T$ with tail index $\alpha$. The entries in italics (for $T=250$ and 1000), make use of the adjustment procedure via multiplicative factor $m\left(T, \alpha, c_{\text {nom }}\right.$ ). The remaining entries do not use the adjustment procedure.

\section{B Determination of $p$-Values for $\tau_{20}$ Test (8)}

Determination of the $p$-value of the $\tau_{20}$ test is done in the same way as for the $D_{T, \kappa}(\mathbf{X})$ test $(7)$ via the MixGAt approximation to the distribution of the test statistic under the null, for a grid of values over various $T$ and $\alpha$. (As this test is only applicable for sample sizes realistically larger than $T=500$, there is less need to use the multiplicative adjustment technique from (23), and thus we omit it.) In 
those simulations, we use $B=20$. The statistic $\tau_{B}(\mathbf{X})$ is then computed from location-adjusted $\mathbf{X}$ (and $B$ can be chosen much larger for a single data set to ensure a nearly unique test statistic), and, for each element in the $T / \alpha$ grid, the associated $p$-value is computed, where it is given as the right tail area greater than the test statistic (i.e., we use a one-sided test, rejecting for large $\tau_{B}$, as we expect the slope coefficient $\hat{b}$ to be positive under the alternative hypothesis). Then, in the same way as for (7), bivariate interpolation is used to deliver the $p$-value, according to the actual sample size $T$ and $\hat{\alpha}_{\text {Hint }}(\mathbf{X})$.

With respect to the null distribution, it was found that, as $\alpha \rightarrow 2$, the shape of the pdf (from kernel density plots based on 10,000 replications) becomes very non-standard, exhibiting spiky behavior, bimodality, and very extreme asymmetry, so that the quality of the MixGAt fits begin to deteriorate. This explains why the actual size of the test, investigated next, is poor for small sample sizes and $\alpha=1.9$.

Analogous to the test (7), we inspect the actual size of the $\tau_{B}(\mathbf{X})$ test (8) for a set of $T$ and $\alpha$. For each replication, we use $B=20$. Table 10 shows the results for nominal sizes $5 \%$ and $1 \%$ (numbers for $10 \%$ were computed, and were qualitatively similar to the $5 \%$ and $1 \%$ cases). The actual and nominal sizes coincide very well for values of $\alpha$ close to 1.5 and/or $T$ large. This is due to the limitations of the interpolation method when $\hat{\alpha}$ is near the border of $(1,2)$, exacerbated for smaller $T$ because of the higher variance of the estimator.

\begin{tabular}{|c|c|c|c|c|c|c|c|c|c|c|}
\hline \multirow{2}{*}{$\begin{array}{l}\text { Nominal } \\
T \backslash \alpha\end{array}$} & \multicolumn{5}{|c|}{0.05} & \multicolumn{5}{|c|}{0.01} \\
\hline & 1.20 & 1.35 & 1.55 & 1.75 & 1.90 & 1.20 & 1.35 & 1.55 & 1.75 & 1.90 \\
\hline 500 & 0.071 & 0.060 & 0.054 & 0.046 & 0.033 & 0.0238 & 0.0132 & 0.0100 & 0.0046 & 0.0040 \\
\hline 750 & 0.060 & 0.056 & 0.052 & 0.044 & 0.036 & 0.0132 & 0.0095 & 0.0104 & 0.0089 & 0.0070 \\
\hline 1000 & 0.060 & 0.055 & 0.053 & 0.045 & 0.035 & 0.0123 & 0.0111 & 0.0102 & 0.0074 & 0.0028 \\
\hline 1500 & 0.058 & 0.055 & 0.055 & 0.047 & 0.033 & 0.0104 & 0.0098 & 0.0096 & 0.0081 & 0.0025 \\
\hline 2500 & 0.055 & 0.050 & 0.051 & 0.053 & 0.039 & 0.0112 & 0.0101 & 0.0109 & 0.0083 & 0.0032 \\
\hline 5000 & 0.053 & 0.051 & 0.050 & 0.054 & 0.050 & 0.0102 & 0.0091 & 0.0099 & 0.0103 & 0.0095 \\
\hline 10000 & 0.048 & 0.048 & 0.052 & 0.053 & 0.048 & 0.0099 & 0.0115 & 0.0108 & 0.0096 & 0.0064 \\
\hline
\end{tabular}

Table 10: Actual sizes of the nominal 5\%, and 1\% $\tau_{20}(\mathbf{X})$ test (8) for data of length $T$ with tail index $\alpha$.

\section{Determination of $p$-Values for ALHADI Test (9)}

We use the same method of approximation as employed for getting the $p$-value of the $D_{T, \kappa}(\mathbf{X})$ test $(7)$ and the $\tau_{B}$ test (8), but we consider use of a two-component MixN (5 parameters) and a two-component mixture of Azzalini (1985) skew-normal (7 parameters), where the skew-normal location-zero, scale-one pdf is given by

$$
f_{\mathrm{SN}}(z ; \lambda)=2 \phi(z) \Phi(\lambda z), \quad \lambda \in \mathbb{R}
$$

where $\phi$ and $\Phi$ are the standard normal pdf and cdf, respectively. Note that it is asymmetric, but not fat-tailed. When $\lambda=0, f_{\mathrm{SN}}$ reduces to the standard normal density; otherwise, it is skewed. We use these instead of the MixGAt, which also fits well, but the estimated degrees of freedom tend be very 
large, and numeric problems during optimization arose occasionally, as it is over-parameterized and not suited for modeling near-Gaussian data. Figure 10 shows four cases, each based on 100,000 replications. For these cases, the matches between the kernel density and the fitted MixGAt, MixN and MixSN are all very good. It appears that the extra flexibility from the MixSN is not necessary, so we use the MixN as the parametric choice for modeling the distribution of the test statistic under the null.

As with the previous two tests, the $p$-values for each entry in a grid of $T$ and $\alpha$ values are computed (but based on the MixN parametric approximation) and bivariate spline interpolation is applied, using the actual $T$ and $\hat{\alpha}_{\text {Hint }}$ in place of the true $\alpha$. Anticipating the same issue which arose with the $D_{T, \kappa}(\mathbf{X})$ test, we use a tighter grid of $\alpha$-values for the $p$-value interpolation construction, $1.05,1.10,1.15, \ldots$, $1.95,1.99$, though we will see this is still not adequate for obtaining the desired accuracy.

The actual sizes of the ALHADI test (using our usual method of $p$-value interpolation, but not using the multiplicative modification method in (23)) are shown in Table 11, for several $T$ and $\alpha$, for nominal sizes $5 \%$ and $1 \%$ (the values for $10 \%$ were also recorded, are qualitatively similar, and not shown). The accuracy of the actual size compared to the nominal is reasonably good for $1.3<\alpha<1.95$ and $T \geqslant 500$, though is poor for $\alpha<1.4$ and $T=250$. The row indicated by $250^{*}$ shows the performance when the true value of $\alpha$ is used (instead of $\hat{\alpha}_{\text {Hint }}$ ) for indexing into the $p$-value table, so that interpolation is not required. The performance for $\alpha=1.2$ and $\alpha=1.35$, with $T=250$, is then much better, showing that the MixN parametric approximation is adequate, and that the problem has to do with the fact that we do not entertain values of $\hat{\alpha}_{\text {Hint }}<1.05$. The row indicated by $5000^{*}$ is similar, and shows that the effect of $p$-value interpolation is still apparent, though far less consequential, even for substantial sample sizes.

We could attempt to deploy the same modification method in (23) for, say, sample sizes $250 \leqslant T \leqslant$ 500 and $1.05 \leqslant \hat{\alpha} \leqslant 1.4$, though more effective would be use of a finer grid of $\alpha$-values, and using values of $\alpha<1$. We omit this because, in our testing applications to financial data, $\hat{\alpha}$ tends to be well above 1.4 , and sample sizes of at least 500 are anyway required to get reasonable power.

\begin{tabular}{|c|c|c|c|c|c|c|c|c|c|c|}
\hline \multirow{2}{*}{$\begin{array}{l}\text { Nominal } \\
T \backslash \alpha\end{array}$} & \multicolumn{5}{|c|}{0.05} & \multicolumn{5}{|c|}{0.01} \\
\hline & 1.20 & 1.35 & 1.55 & 1.75 & 1.90 & 1.20 & 1.35 & 1.55 & 1.75 & 1.90 \\
\hline 250 & 0.0963 & 0.0617 & 0.0434 & 0.0431 & 0.0549 & 0.0436 & 0.0203 & 0.0075 & 0.0063 & 0.0091 \\
\hline $250^{*}$ & 0.0532 & 0.0501 & 0.0525 & 0.0477 & 0.0504 & 0.0095 & 0.0096 & 0.0101 & 0.0098 & 0.0090 \\
\hline 500 & 0.0837 & 0.0501 & 0.0441 & 0.0455 & 0.0551 & 0.0342 & 0.0120 & 0.0068 & 0.0068 & 0.0102 \\
\hline 1000 & 0.0665 & 0.0479 & 0.0459 & 0.0473 & 0.0556 & 0.0211 & 0.0097 & 0.0073 & 0.0075 & $0.010 s$ \\
\hline 2500 & 0.0501 & 0.0438 & 0.0461 & 0.0472 & 0.0555 & 0.0122 & 0.0076 & 0.0076 & 0.0081 & 0.0105 \\
\hline 5000 & 0.0468 & 0.0453 & 0.0484 & 0.0495 & 0.0546 & 0.0093 & 0.0100 & 0.0088 & 0.0089 & 0.0118 \\
\hline $5000 *$ & 0.0502 & 0.0502 & 0.0496 & 0.0501 & 0.0535 & 0.0092 & 0.0110 & 0.0098 & 0.0098 & 0.0097 \\
\hline
\end{tabular}

Table 11: Actual sizes of the nominal $5 \%$ and $1 \% A(\mathbf{X})$ test (9) for data of length $T$ with tail index $\alpha$. The rows labeled $250^{*}$ and $5000^{*}$ indicate the use of the true value of $\alpha$ instead of using $\hat{\alpha}_{\text {Hint }}$ and linear interpolation into the constructed table of $p$-values. 

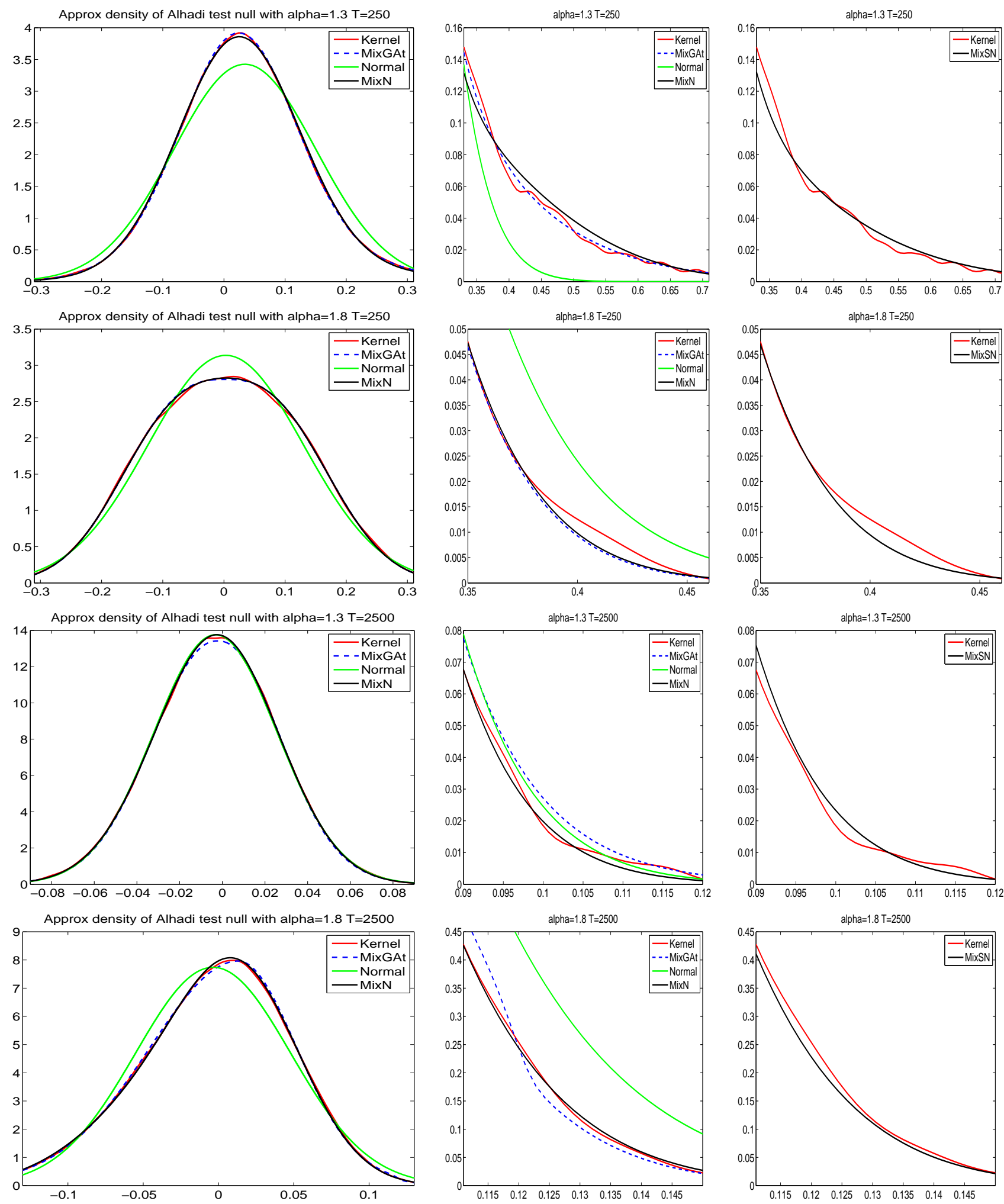

Figure 10: Left: Kernel density, fitted normal, MixN, and MixGAt, for 100,000 simulated realizations of the $A(\mathbf{X})$ test statistic (9) under the null, for the indicated sample sizes $T$ and values of $\alpha$.

Middle: Same as left, but magnified view of right tail.

Right: Same as middle, but showing the fit of the two-component mixture of skew-normal distribution. 


\section{Study of Combined $A+D$ Test}

For the combined $A+D$ test, we show the power results using the Student's $t$ and NIG alternatives, based on the same parameters as were used in Tables 2 and 4, respectively, but adding some larger values of the NIG shape parameter $\alpha$. Furthermore, we try this with the smaller sample sizes $T=250$ and $T=500$ (as opposed to 500, 1000 and 2500, as used in Tables 2 to 5) because (i) both ALHADI and $D_{T, 5}$ are applicable for smaller sample sizes, as opposed to the summability-based test $\tau_{20}$, and (ii) it would be useful to have a test with higher power for smaller sample sizes.

\begin{tabular}{|c|c|c|c|c|c|c|c|c|c|c|c|c|c|}
\hline \multicolumn{2}{|c|}{ Sample size } & \multicolumn{6}{|c|}{$T=250$} & \multicolumn{6}{|c|}{$T=500$} \\
\hline Test & Eq. & 1.5 & 2 & 3 & 4 & 5 & 6 & 1.5 & 2 & 3 & 4 & 5 & 6 \\
\hline$A$ & (9) & 0.14 & 0.17 & 0.20 & 0.19 & 0.18 & 0.14 & 0.18 & 0.29 & 0.39 & 0.33 & 0.26 & 0.22 \\
\hline$D_{T, 5}$ & (7) & 0.12 & 0.09 & 0.08 & 0.09 & 0.08 & 0.06 & 0.12 & 0.12 & 0.12 & 0.11 & 0.08 & 0.10 \\
\hline$A+D$ & & 0.11 & 0.14 & 0.17 & 0.18 & 0.17 & 0.17 & 0.14 & 0.22 & 0.30 & 0.26 & 0.19 & 0.18 \\
\hline
\end{tabular}

Table 12: Power of the ALHADI, $D_{T, 5}$, and combined test $A+D$, against the Student's $t$ alternative, for degrees of freedom values $v=1.5,2,3,4,5,6$, for the nominal size of $5 \%$.

Table 12 shows the results against the Student's $t$, based on 1000 replications. We see that, only in one case (for $T=250$ and six degrees of freedom) does the combined test have (slightly) higher power than the ALHADI test. Table 13 shows the results against the NIG, based on 1000 replications. We see that, only in one case (for $T=250$ and NIG shape parameter $\alpha=1.5$ ) does the combined $A+D$ test outperform the ALHADI component, and not by much. For the MixN alternative (results not shown), the $A+D$ test was found to be less powerful than ALHADI for all constellations considered, for both sample sizes $T=250$ and $T=500$.

\begin{tabular}{|c|c|c|c|c|c|c|c|c|c|c|c|c|c|c|c|}
\hline \multicolumn{2}{|c|}{ Sample size } & \multicolumn{7}{|c|}{$T=250$} & \multicolumn{7}{|c|}{$T=500$} \\
\hline Test & Eq. & 0.3 & 0.6 & 0.9 & 1.2 & 1.5 & 1.8 & 2.1 & 0.3 & 0.6 & 0.9 & 1.2 & 1.5 & 1.8 & 2.1 \\
\hline$A$ & (9) & 0.43 & 0.38 & 0.30 & 0.23 & 0.22 & 0.17 & 0.19 & 0.79 & 0.69 & 0.55 & 0.45 & 0.40 & 0.33 & 0.30 \\
\hline$D_{T, 5}$ & (7) & 0.16 & 0.11 & 0.10 & 0.09 & 0.10 & 0.07 & 0.07 & 0.21 & 0.19 & 0.14 & 0.12 & 0.12 & 0.11 & 0.12 \\
\hline$A+I$ & & 0.34 & 0.29 & 0.27 & 0.23 & 0.24 & 0.20 & 0.19 & 0.65 & 0.56 & 0.44 & 0.36 & 0.30 & 0.27 & 0.24 \\
\hline Avera & $\hat{\alpha}_{\text {Hint }}$ & 1.54 & 1.66 & 1.73 & 1.77 & 1.80 & 1.82 & 1.84 & 1.53 & 1.66 & 1.72 & 1.76 & 1.80 & 1.82 & 1.84 \\
\hline
\end{tabular}

Table 13: Power of the ALHADI, $D_{T, 5}$, and combined test $A+D$, against the NIG alternative, with pdf (10), using $\beta=0, \mu=0, \delta=1$, and shape values $\alpha=0.3,0.6,0.9,1.2,1.5,1.8,2.1$, for the nominal size of $5 \%$.

We conjecture that the reason for this poor performance is that the $D_{T, 5}$ test is so much less powerful than ALHADI (for these alternatives at least), that it weakens the test. It appears that combining two (not perfectly correlated) tests that have roughly equal power is ideal, as with the $A+\tau_{20}$ test.

\section{Acknowledgements}

Financial support by the Swiss National Science Foundation (SNSF) through project \#150277 is gratefully acknowledged. The author wishes to thank J Huston McCulloch, Michael Wolf, three anonymous 
referees, and the editor Erricos Kontoghiorghes for insightful comments that led to a substantial improvement of this paper.

\section{References}

Azzalini, A. (1985). A Class of Distributions which Includes the Normal Ones. Scandinavian Journal of Statistics, 12:171-178.

Borak, S., Härdle, W., and Weron, R. (2005). Stable Distributions. In Čížek, P., Härdle, W., and Weron, R., editors, Statistical Tools for Finance and Insurance, pages 21-44. Springer Verlag.

Broda, S. and Paolella, M. S. (2007). Saddlepoint Approximations for the Doubly Noncentral $t$ Distribution. Computational Statistics and Data Analysis, 51:2907-2918.

Broda, S. A., Haas, M., Krause, J., Paolella, M. S., and Steude, S. C. (2013). Stable Mixture GARCH Models. Journal of Econometrics, 172(2):292-306.

Broda, S. A. and Paolella, M. S. (2009). CHICAGO: A Fast and Accurate Method for Portfolio Risk Calculation. Journal of Financial Econometrics, 7(4):412-436.

Brooks, C., Clare, A. D., Dalle-Molle, J. W., and Persand, G. (2005). A Comparison of Extreme Value Theory Approaches for Determining Value at Risk. Journal of Empirical Finance, 12(2):339-352.

Chambers, J. M., Mallows, C. L., and Stuck, B. W. (1976). A Method for Simulating Stable Random Variables. Journal of the American Statistical Association, 71:340-344.

Choi, P. and Nam, K. (2008). Asymmetric and Leptokurtic Distribution for Heteroscedastic Asset Returns: The $S_{U}$-Normal Distribution. Journal of Empirical Finance, 15:41-63.

Doganoglu, T. and Mittnik, S. (1998). An Approximation Procedure for Asymmetric Stable Paretian Densities. Computational Statistics, 13:463-475.

Fama, E. and Roll, R. (1971). Parameter Estimates for Symmetric Stable Distributions. Journal of the American Statistical Association, 66(334):331-338.

Fama, E. F. (1963). Mandelbrot and the Stable Paretian Hypothesis. Journal of Business, 36:420-429.

Ghose, D. and Kroner, K. F. (1995). The Relationship between GARCH and Symmetric Stable Processes: Finding the Source of Fat Tails in Financial Data. Journal of Empirical Finance, 2:225-251.

Groenendijk, P. A., Lucas, A., and de Vries, C. G. (1995). A Note on the Relationship between GARCH and Symmetric Stable Processes. Journal of Empirical Finance, 2:253-264.

Haas, M., Krause, J., Paolella, M. S., and Steude, S. C. (2013). Time-Varying Mixture GARCH Models and Asymmetric Volatility. North American Journal of Economics and Finance, 26:602-623.

Haas, M., Mittnik, S., and Paolella, M. S. (2004). Mixed Normal Conditional Heteroskedasticity. Journal of Financial Econometrics, 2(2):211-250.

Halvarsson, D. (2013). On the Estimation of Skewed Geometric Stable Distributions. Working paper No. 216, The Royal Institute of Technology, Division of Economics, Stockholm, Sweden.

Harvey, C. R. and Siddique, A. (1999). Autoregressive Conditional Skewness. Journal of Financial and Quantitative Analysis, 34(4):465-487.

Heyde, C. C. and Kou, S. G. (2004). On the Controversy over Tailweight of Distributions. Operations Research Letters, 32:399-408. 
Hill, B. M. (1975). A Simple General Approach to Inference About the Tail of a Distribution. Annals of Statistics, 3(5):1163-1174.

Johnson, N. L. (1949). Systems of Frequency Curves Generated by Method of Translation. Biometrika, $36: 149-176$.

Jondeau, E. (2010). Asymmetry in Tail Dependence of Equity Portfolios. National Centre of Competence in Research, Financial Valuation and Risk Management, Working Paper No. 658.

Jondeau, E., Poon, S.-H., and Rockinger, M. (2007). Financial Modeling Under Non-Gaussian Distributions. Springer Verlag, London.

Jones, M. C. and Faddy, M. J. (2003). A Skew Extension of the $t$ Distribution with Applications. Journal of the Royal Statistical Society, Series B, 65:159-174.

Kotz, S. and Nadarajah, S. (2004). Multivariate t Distributions and Their Applications. Cambridge University Press.

Koutrouvelis, I. A. and Meintanis, S. G. (1999). Testing for Stability Based on the Empirical Characteristic Function with Applications to Financial Data. Journal of Statistical Computation and Simulation, 64(4):275-300.

Kozubowski, T. J. (2000). Exponential Mixture Representation of Geometric Stable Distributions. Annals of the Institute of Statistical Mathematics, 52(2):231-238.

Kozubowski, T. J. and Rachev, S. T. (1999). Univariate Geometric Stable Laws. Journal of Computational Analysis and Applications, 1(2):177-217.

Krause, J. and Paolella, M. S. (2014). A Fast, Accurate Method for Value at Risk and Expected Shortfall. Econometrics, 2:98-122.

Kshirsagar, A. M. (1961). Some Extensions of the Multivariate Generalization $t$ distribution and the Multivariate Generalization of the Distribution of the Regression Coefficient. Proceedings of the Cambridge Philosophical Society, 57:80-85.

Küchler, U. and Tappe, S. (2013). Tempered Stable Distributions and Processes. Stochastic Processes and their Applications, 123(12):4256-4293.

Lau, A. H.-L., Lau, H.-S., and Wingender, J. R. (1990). The Distribution of Stock Returns: New Evidence Against the Stable Model. Journal of Business and Economic Statistics, 8:217-223.

Lau, H.-S. and Lau, A. H.-L. (1993). The Reliability of the Stability-Under-Addition Test for the Stable-Paretian Hypothesis. Journal of Statistical Computation and Simulation, 48:67-80.

Loretan, M. and Phillips, P. C. B. (1994). Testing the Covariance Stationarity of Heavy-Tailed Time Series. Journal of Empirical Finance, 1:211-248.

Lye, J. N. and Martin, V. L. (1993). Robust Estimation, Nonnormalities, and Generalized Exponential Distributions. Journal of the American Statistical Association, 88(421):261-267.

Mandelbrot, B. (1963). The Variation of Certain Speculative Prices. The Journal of Business, $36(4): 394-419$.

Matsui, M. and Takemura, A. (2008). Goodness-of-Fit Tests for Symmetric Stable DistributionsEmpirical Characteristic Function Approach. TEST, 17(3):546-566.

McCulloch, J. H. (1986). Simple Consistent Estimators of Stable Distribution Parameters. Communications in Statistics - Simulation and Computation, 15(4):1109-1136. 
McCulloch, J. H. (1997a). Financial Applications of Stable Distributions. In Maddala, G. and Rao, C., editors, Handbook of Statistics, volume 14. Elsevier Science, Amsterdam.

McCulloch, J. H. (1997b). Measuring Tail Thickness in Order to Estimate the Stable Index $\alpha$ : A Critique. Journal of Business and Economic Statistics, 15(1):74-81.

McCulloch, J. H. and Percy, E. R. (2013). Extended Neyman Smooth Goodness-of-Fit Tests, Applied to Competing Heavy-Tailed Distributions. Journal of Econometrics, 172(2):275-282.

McNeil, A. J., Frey, R., and Embrechts, P. (2005). Quantitative Risk Management: Concepts, Techniques, and Tools. Princeton University Press, Princeton.

Meintanis, S. G. (2005). Consistent Tests for Symmetric Stability with Finite Mean Based on the Empirical Characteristic Function. Journal of Statistical Planning and Inference, 128:373-380.

Mittnik, S., Doganoglu, T., and Chenyao, D. (1999). Computing the Probability Density Function of the Stable Paretian Distribution. Mathematical and Computer Modelling, 29:235-240.

Mittnik, S. and Paolella, M. S. (1999). A Simple Estimator for the Characteristic Exponent of the Stable Paretian Distribution. Mathematical and Computer Modelling, 29:161-176.

Mittnik, S., Paolella, M. S., and Rachev, S. T. (1998). A Tail Estimator for the Index of the Stable Paretian Distribution. Communications in Statistics-Theory and Methods, 27(5):1239-1262.

Mittnik, S., Paolella, M. S., and Rachev, S. T. (2000). Diagnosing and Treating the Fat Tails in Financial Returns Data. Journal of Empirical Finance, 7:389-416.

Nolan, J. P. (1997). Numerical Calculation of Stable Densities and Distribution Functions. Communications in Statistics: Stochastic Models, 13(4):759-774.

Nolan, J. P. (1999). Fitting Data and Assessing Goodness-of-fit with Stable Distributions.

Nolan, J. P. (2016). Stable Distributions - Models for Heavy Tailed Data. Birkhäuser, Boston. forthcoming; Chapter 1 online.

Paolella, M. S. (2001). Testing the Stable Paretian Assumption. Mathematical and Computer Modelling, 34:1095-1112.

Paolella, M. S. (2007). Intermediate Probability: A Computational Approach. Wiley \& Sons, Chichester, West Sussex, England.

Paolella, M. S. (2013). Multivariate Asset Return Prediction with Mixture Models. European Journal of Finance, pages 1-39.

Paolella, M. S. (2015). New Graphical Methods and Test Statistics for Testing Composite Normality. Econometrics, 3:532-560.

Paolella, M. S. (2016). Stable-GARCH Models for Financial Returns: Fast Estimation and Tests for Stability. Forthcoming in: Econometrics.

Platen, E. and Heath, D. (2006). A Benchmark Approach to Quantitative Finance. Springer, New York.

Rachev, S. T. and Mittnik, S. (2000). Stable Paretian Models in Finance. Wiley \& Sons, Chichester, West Sussex, England.

Samorodnitsky, G. and Taqqu, M. S. (1994). Stable Non-Gaussian Random Processes, Stochastic Models with Infinite Variance. Chapman \& Hall, London.

Vuong, Q. H. (1989). Likelihood Ratio Tests for Model Selection and Non-Nested Hypotheses. Econometrica, 57(2):307-333. 